\title{
INFORMATION AND ENTROPY - TOP-DOWN OR BOTTOM-UP DEVELOPMENT IN LIVING SYSTEMS?
}

\author{
A.C. McINTOSH \\ Energy and Resources Research Institute, University of Leeds, Leeds, UK.
}

Editor's Note: This paper presents a different paradigm than the traditional view. It is, in the view of the Journal, an exploratory paper that does not give a complete justification for the alternative view. The reader should not assume that the Journal or the reviewers agree with the conclusions of the paper. It is a valuable contribution that challenges the conventional vision that systems can design and organise themselves. The Journal hopes that the paper will promote the exchange of ideas in this important topic. Comments are invited in the form of 'Letters to the Editor'.

\section{ABSTRACT}

This paper deals with the fundamental and challenging question of the ultimate origin of genetic information from a thermodynamic perspective. The theory of evolution postulates that random mutations and natural selection can increase genetic information over successive generations. It is often argued from an evolutionary perspective that this does not violate the second law of thermodynamics because it is proposed that the entropy of a non-isolated system could reduce due to energy input from an outside source, especially the sun when considering the earth as a biotic system. By this it is proposed that a particular system can become organised at the expense of an increase in entropy elsewhere. However, whilst this argument works for structures such as snowflakes that are formed by natural forces, it does not work for genetic information because the information system is composed of machinery which requires precise and non-spontaneous raised free energy levels - and crystals like snowflakes have zero free energy as the phase transition occurs. The functional machinery of biological systems such as DNA, RNA and proteins requires that precise, non-spontaneous raised free energies be formed in the molecular bonds which are maintained in a far from equilibrium state. Furthermore, biological structures contain coded instructions which, as is shown in this paper, are not defined by the matter and energy of the molecules carrying this information. Thus, the specified complexity cannot be created by natural forces even in conditions far from equilibrium. The genetic information needed to code for complex structures like proteins actually requires information which organises the natural forces surrounding it and not the other way around - the information is crucially not defined by the material on which it sits. The information system locally requires the free energies of the molecular machinery to be raised in order for the information to be stored. Consequently, the fundamental laws of thermodynamics show that entropy reduction which can occur naturally in non-isolated systems is not a sufficient argument to explain the origin of either biological machinery or genetic information that is inextricably intertwined with it. This paper highlights the distinctive and non-material nature of information and its relationship with matter, energy and natural forces. It is proposed in conclusion that it is the non-material information (transcendent to the matter and energy) that is actually itself constraining the local thermodynamics to be in ordered disequilibrium and with specified raised free energy levels necessary for the molecular and cellular machinery to operate.

Keywords: entropy, evolution, free energy, information, intelligent design, machines, non-isolated systems.

\section{INTRODUCTION}

How does information relate to the hardware used? Is it possible to measure information and its effect on the local thermodynamics of the system hardware? How do these relate in real systems, and can one connect and quantify the effect of information on matter and energy? This issue is most importantly considered in the realm of living systems, where one quickly becomes aware of the extraordinary complexity which so organises the biochemical proteins at the molecular level as to effectively build digital machinery that for many years, since the discovery of the DNA model by Crick and Watson [1], has been the goal of modern software engineers to emulate.

Genetic information in the form of DNA is responsible for defining the vast array of biological structures and processes that we see around us in the natural world. In the human genome, there are said to be around three billion units of information. These units specify the structures and processes 
that take place in the human body. The major function of DNA is to specify proteins. Proteins consist of long polymer chains of amino acids and there are many thousands of different types of them. Proteins include structures like collagen (used in tendons and ligaments), enzymes, hormones and blood, and have chains with very complicated three-dimensional structures. To claim that all proteins have evolved from other proteins is very bold. A particular protein might have hundreds of physical differences with its closest counterpart.

It is well known that genetic information is modified from one generation to the next by sexual recombination. This means that it is possible for natural selection or selective breeding to emphasise certain features such as high speed in a horse or the aerodynamic prowess of the wandering albatross. However, since sexual recombination involves shuffling of existing genes, it cannot of itself produce new information.

Therefore, it has been argued that gene mutations have the potential to create new genetic information in the gene pool. However, a crucial question is whether random mutations can really generate the type of complex information needed to specify a complex protein? Can such mutations produce new machinery which was not there in principle or latently coded beforehand? Though superficially it seems that a non-isolated system could have the potential to increase information by local entropy decrease, the argument developed in this paper is that this does not work for biological information systems since they involve specified complexity that cannot fully be created by natural forces. Just because some structures can be generated automatically in nature (like crystals which do not have any increase in free energy as the change of state occurs), it does not mean that the principle can be extrapolated to assume that any amount of complexity can be produced in nature by such means.

The functional complexity of biological systems is clearly heavily dependent on the material environment in which such a system is operating, and indeed applies all the same chemical and physical laws that are used to such good effect by any man-made machine. What though are the laws that such organisation must inherently obey for natural systems? Can one quantify the organisational structure which sits on top of the matter and energy in any real system?

In the next section, a review is made of the various approaches that have been proposed concerning how a localised increase in order could be obtained and it also summarises the arguments of the key proponents of this theory. The subsequent sections present three fundamental concepts of thermodynamics which show that entropy reduction in an open system is not a sufficient argument to explain the origin of genetic information. These are the fundamentals of energy flow behind the second law of thermodynamics, the definition of a machine and the definition of information itself.

This paper highlights the importance of information, its relationship with matter and energy, and proposes a 'top-down' approach for the understanding of the information - machinery interface.

\section{REVIEW OF EXISTING THEORIES OF INFORMATION}

Some aspects of this paper were discussed in a conference paper of similar title presented at the third Design and Nature Conference in 2006 [2]. In that paper, the author considered the fundamental aspects of entropy and the second law of thermodynamics applied first of all in the traditional definitions used in heat and chemical systems. Then analogous representations of so-called 'logical entropy' were discussed (particularly the work of Prigogine $[3,4]$ ) in the context of attempts to simulate in a rational way the idea of functional complexity emerging from simpler life forms. Prigogine's work primarily sought to express self-organisation in terms of non-equilibrium thermodynamics and the term 'Prigogine entropy' was introduced. The basic thesis of all these arguments is that in a non-isolated system far from equilibrium, a local lowering in entropy (and consequently a rise in ordered structure) can take place at the expense of increasing the entropy in the rest of the non-isolated system. However, even though Prigogine et al. [5] acknowledged that 
'in a non-isolated system there exists a possibility for formation of ordered, low-entropy structures at sufficiently low temperatures. This ordering principle is responsible for the appearance of ordered structures such as crystals as well as the phenomena of phase transitions',

he ends this discussion by saying,

'Unfortunately this principle cannot explain the formation of biological structures'.

Nevertheless, Prigogine's argument is further developed [6] into considering non-equilibrium open systems (allowing mass transfer as well as thermodynamic transfer across the boundary) on the basis of entropy exchange with the surroundings. Much has been written by other authors seeking to justify this approach of bringing in an ordering principle which is either a product of the material itself in some way (e.g. Mayr [7], Maynard Smith and Szathmary [8] and Ball [9]) or seeking a non-material organising principle in some way which will then quantify the lowering locally of entropy at the expense of increasing entropy elsewhere (of particular note are the works of Wicken [10], Kauffman [11] and Brooks and Wiley [12] in this approach). The idea is that though physical processes are essentially dissipative, in an unsteady system a step up in the temporary feeding in of negative entropy will enable the overall system to ascend to a new quasi-steady state. Prigogine's work is considered further in Section 5.2.2.

A paper by Martyushev and Seleznev [13] has brought a lot of these ideas together under an overarching principle called the 'maximum entropy production principle' (MEPP), whereby the authors argue that any non-equilibrium system will always seek to maximise the entropy around it, whilst maintaining its own low entropy locally. This is an important extension of the equilibrium thermodynamic principle - viz. that all reactions will tend to maximise entropy and fall away from a free energy state. Whilst this statement of the MEPP undoubtedly holds true, it somewhat begs the question as to how a local system can possibly reduce the entropy without existing machinery to do this. It is this last matter which concerns us in this paper. How can local organisational structures arise which were not in principle existing beforehand? Furthermore, how can information systems develop, and what are the local thermodynamic implications of such systems?

In order to move into discussing these questions, an important issue needs addressing in order to show why this discussion is so fundamental. Many had thought that Shannon's work on data transmission could be the answer all were seeking to give rise to new local structures. Shannon's work [14] is essentially a theory for the transmission of data. The concept of entropy in information theory describes how much information there is in a signal or event. And Shannon entropy provides a lower bound for the space occupied in transmission by the coding of this data representation. This is called 'data compression' and became of great importance in the development of data transfer after World War II. Allied closely to this is the concept of the definition of information which though some had thought could be simply reduced to Shannon ideas of the entropy, it became clear that the true information content of a signal is far more than the simple recipe of Shannon's theory which essentially only deals with the transmission of existing data.

Dawkins referring to the Shannon concepts in an essay entitled 'The Information Challenge' [15] made the following statement which is quoted in full since it lies right at the heart of the thesis held by most evolutionary biologists that information increase is possible by natural selection operating on successive mutations:

Let me turn, finally to another way of looking at whether the information content of genomes increases evolution. We now switch from the broad sweep of evolutionary history to the minutiae of natural selection. Natural selection itself, when you think about it, is a narrowing down from a wide initial field of possible alternatives, to the narrower field of the alternatives actually chosen. Random genetic error (mutation), sexual recombination and migratory mixing all provide a wide 
field of genetic variation: the available alternatives. Mutation is not an increase in true information content, rather the reverse, for mutation in the Shannon analogy, contributes to increasing the prior uncertainty. But now we come to natural selection, which reduces the 'prior uncertainty' and therefore, in Shannon's sense, contributes information to the gene pool. In every generation, natural selection removes the less successful genes from the gene pool, so the remaining gene pool is a narrower subset. The narrowing is non-random, in the direction of improvement, where improvement is defined, in the Darwinian way, as improvement in fitness to survive and reproduce. Of course the total range of variation is topped up again in every generation by new mutation and other kinds of variation. But it still remains true that natural selection is a narrowing down from an initially wider field of possibilities, including unsuccessful ones, to a narrower field of successful ones. This is analogous to the definition of information with which we began: information is what enables the narrowing down from prior uncertainty (the initial range of possibilities) to later certainty (the "successful' choice among prior probabilities). According to this analogy, natural selection is by definition a process whereby information is fed into the gene pool of the next generation. ([15], pp. 120-121)

He refers to 'true information content' and later to a definition of information as that which 'enables the narrowing down from prior uncertainty'. Mutation is not an increase in information content, but in his thinking, natural selection coupled with an increase in possible mutation 'options' causes new biological function to emerge. New information (which for Dawkins simply becomes a synonym for Darwinian 'improvement in fitness to survive') is added by natural selective processes. Even in this materialistic definition of information (as against a non-material definition, viz. that DNA code is a parallel to software in computers) such statements by Dawkins raise issues concerning the thermodynamics of what he is claiming to be possible. Can new biological functions emerge spontaneously which were not latently there already (as sub-functions or in embryo form coded for by a DNA template)? The answer to these questions is not just of academic importance but lies at the heart of the origins debate - a debate which has major philosophical implications. The ultimate question in origins must be: Can information increase in a purely materialistic or naturalistic way? It is not satisfactory to simply assume that information has to have arisen in this way. The alternative of original design must be allowed and all options examined carefully.

Dawkins in his own paradigm of reality is ruthlessly consistent but, as we shall see, hits a thermodynamic impossibility in this materialist approach. He has assumed mutations increase information content but it becomes evident that thermodynamically this is not true. It needs to be acknowledged that there are others who whilst resisting the rampant materialism of Dawkins referred to earlier, see the work of answer to lie in some form of cosmic entropy balance. Clearly, Prigogine [3-6] was of this view and the work of Wicken [10], Kauffman [11] and Brooks and Wiley [12] is in a similar vein. Goonatilake [16] refers to the flow of information as a quantity which, though not material, is associated with the arrangement of matter and comes from a self-organising process yet to be understood. Kauffman similarly appeals to such a concept and Brooks and Wiley [12] seek to quantify such a flow of information in terms of non-equilibrium thermodynamics. Such approaches are also there in other disciplines. So, for example, the astronomer Paul Davies in his book, The Fifth Miracle - The Search for the Origin of Life [17] has the same concept in his second chapter, 'Against the tide'. Under the section 'Where does biological information come from?' he again appeals to a flow of information by stating, 'The source of biological information, then, is the organism's environment' ( $\mathrm{p}$. 33). In particular, he specifically appeals to gravity as being the mechanism for what he terms the 'fountainhead of all order' regarding gravity as a source of 'information' (by which it is taken he means structure) in that it causes regions of different density to appear in the Universe. However whether this really solves the information gap is questionable since information (as we shall explore) is far more than simply inhomogeneity in temperature and 
density. Murray [18] has done remarkable work showing that pattern formation can be mathematically predicted from the nonlinear reaction-diffusion equations which govern many systems far from equilibrium, and Peter Coveney and Roger Highfield (science editor of the Daily Telegraph) picked up a similar theme with their well-acclaimed book The Arrow of Time [19], labelling such processes as 'creative evolution' (see their chapter 6, p. 182). A good summary of many of these approaches is to be found in Collins et al. [20] and also in Chaisson [21]. These authors are all of the view that there is a cosmic flow of information from a source either in gravity or in some form of pattern formation which carries with it function and generates specified complexity. However, how much of this conclusion is really a product of the naturalistic paradigm used is a matter of debate. In reality, the paradigm being used precludes the notion that information may actually be a product of an intelligent source.

The problem with all these approaches is that whilst they admit to the presence of a non-material concept of arrangement (and in that sense are not purely materialistic - what I term later the 'bottom-up' approach), they are, however eruditely, in the end appealing to a notion that somehow there will be found a principle, perhaps a yet-to-be-found new principle of thermodynamics, that will explain such flow of Information/order/low entropy.

These advocates are often quick to point out that they do not view the materialistic approach to be correct, but are also very much against a third option which is perfectly valid but requires a complete change of paradigm - that is, it requires one to accept that intelligence might actually be involved rather more directly in setting up functional arrangements/patterns/order to start with. In the view of the author there is a perfectly consistent view which is a top-down approach where biological information already present in the phenotypic creature (and not emergent as claimed in the traditional bottom-up approach) constrains the system of matter and energy constituting the living entity to follow intricate non-equilibrium chemical pathways. These pathways whilst obeying all the laws of thermodynamics are constantly supporting the coded software which is present within them such as in the DNA held in the cells of all living systems and also in the information now coming to light in the very complex three-dimensional folded protein structures which are also found within the cell. It is this thesis of a top-down approach which is explored in this work.

Closely related to these considerations is the philosophical issue of epistemology - the fundamental way by which reality itself is interpreted. No scientist looking at these concepts can really escape this underlying issue. Indeed the very rewarding read of Penrose's classic writing The Emperor's New Mind [22] is closely related to the concepts that the author is grappling with in this paper. Whilst he appeals to a cosmic flow of entropy (see, for example, his discussion in pp. 411-417, The Origin of Low Entropy in the Universe in chapter 7 'Cosmology and the arrow of time') he does very much withstand the notion that intelligence will be reproducible in robotic terms. His view of reality as being essentially Platonic (see p. 151 in particular of his tour de force chapter 4 'Truth, proof and insight') rules out straight materialism, and he brilliantly discounts intelligence as being algorithmic in nature. Not all would agree with his dualistic view of nature, but he nevertheless shows that in particular the concept of mind (the main theme of his book of course) cannot be begun to be understood in terms of existing science. Recently, James Le Fanu (a well-known columnist in the Daily Telegraph and a medical doctor) has written [23]

That dual nature of reality, the material and the non-material, would remain buried so long as science continued to advance towards that better future. But when its remorseless progress reached its final destination, the genome of man and fly spelt out, the brain observed 'in action' smelling a rose and constructing a sentence, the reality of the non-material domain would, inevitably, re-emerge from the shadows.

The realm of philosophy to which such a discussion takes us is beyond the strict scope of this paper which shows that one cannot really address the science itself without acknowledging that there 
are major philosophical assumptions that need to be admitted in all viewpoints. The major issue is that the evidence should be allowed to be put without prejudging the best fitting paradigm.

\section{THE PRINCIPLE OF ENERGY FLOW - THE SECOND LAW OF THERMODYNAMICS}

It is impossible, by means of inanimate material agency, to derive mechanical effect from any portion of matter by cooling it below the temperature of the coldest of the surrounding objects.

So wrote William Thomson (Lord Kelvin) in his seminal work [24] where he first explored (along with Clausius, Joule, Carnot and others) what would become known as the principles, the laws of thermodynamics. Joule had already shown [25] the equivalence of mechanical energy and heat in terms of one being possible to convert into the other. Now Lord Kelvin was seeking to bring to a succinct statement the findings of Carnot that [26] 'no engine whatever, with source and refrigerator at the same temperatures, can get more work from a given quantity of heat introduced than any engine which satisfies the condition of reversibility .... This led to the notion that there is always a maximum amount of work that can be done by any isolated system. With the additional observation that there was always wastage in any energy transfer, this then led to the enunciation of the main principles of thermodynamics that we have today.

A precise modern statement of the second law of thermodynamics is: 'The amount of energy available for useful work in a given isolated system is decreasing. The entropy (dissipated useful energy per degree Kelvin) is always increasing' [2].

Essentially this states that there is no 'free lunch'. The statement of course precludes the movement of heat and work and mass crossing the boundary. However, even in an open system, where mass, heat and work can cross the boundary of the system, the principle of the flow of energy using the fastest path to equilibrium will hold. Essentially, heat always flows from hot to cold. In any process or cycle, heat can be made to do work but always some energy will be lost to the environment, and that energy cannot be retrieved. The work may be as simple as driving a piston in a pump to bring water to the surface, or as complicated as a gas turbine powering many aircrafts today. Some energy will always be lost which cannot be retrieved within the same system. A simple illustration would be of a hydroelectric plant. Water flows downhill and loses potential energy which is changed into kinetic energy. This can again be made to do work, but some energy will be lost such that if one was to use all the energy generated to pump the same water back up to its source, it would not reach the same level. The difference of original potential energy to that corresponding to the new level, divided by the temperature (which in that case is virtually constant) is the entropy of the system. Such a measure will always give an entropy gain.

In a second seminal paper, Thomson (Lord Kelvin) [27] set forth some major principles which followed from the laws of thermodynamics that he, Carnot and others had studied. He stated first:

The object of the present communication is to call attention to the remarkable consequences which follow from Carnot's proposition, that there is an absolute waste of mechanical energy available to man when heat is allowed to pass from one body to another at a lower temperature, by any means not fulfilling his criterion of a 'perfect thermo-dynamic engine', established, on a new foundation, in the dynamical theory of heat. As it is most certain that Creative Power alone can either call into existence or annihilate mechanical energy, the 'waste' referred to cannot be annihilation, but must be some transformation of energy.

He then went on to enunciate the following three propositions which logically follow from the universally applicable second law of thermodynamics:

1. There is present in the material world a universal tendency to the dissipation of mechanical energy.

2. Any restoration of mechanical energy, without more than an equivalent of dissipation, is impossible in inanimate material processes, and is probably never effected by means 
of organic matter, either endowed with vegetable life or subjected to the will of an animated creature.

3. Within a finite period of time past, the earth must have been, and within a finite period of time to come, the earth must again be unfit for the habitation of man as at present constituted, unless operations have been or are to be performed which are impossible under the laws to which the known operations going on at present in the world are subject.

Put in modern terms, the fundamental nature of the second law was being expounded by Lord Kelvin's propositions [28]. There is no known system where the second law of thermodynamics does not apply. The fact that the entropy of a given isolated system increases, effectively brings with it an inevitable decline in usefulness of all systems. The phrase 'arrow of time' [19] is often used to describe this, since the second law brings in the concept of non-reversibility of all real systems. Whilst it is true that the third statement does not overtly take account of highly non-linear changes in the Universe, as a general statement that the Universe as a whole must be tending to a state of increased entropy, it is correct.

For a given system, the change in the internal energy $E$ and the work done by the system $W$ will for a perfect exchange be balanced with the amount of work achieved, being exactly balanced by the drop in internal energy. That this is not the case in real systems is due to heat $Q$ being dissipated by the system.

The first law of thermodynamics is a statement of the conservation of energy principle. Thus,

$$
d E+d W=d Q .
$$

For a general system the most fundamental statement of the second law of thermodynamics is the Clausius statement that 'The sum of all the ratios $d Q / T$ for a cyclic process is always negative or zero'.

Mathematically, this can be stated as

$$
\oint \frac{d Q}{T} \leq 0
$$

The entropy of a system is defined as

$$
d S=\left(\frac{d Q}{T}\right)_{\text {int rev }}\left(\mathrm{kJ} \mathrm{K}^{-1}\right) .
$$

where the subscript 'int rev' means an internally reversible process and temperature $T$ is measured in degrees Kelvin (K). The change in entropy $S$ is thus defined along a reversible path, and is the change in heat dissipated per unit temperature. Entropy is in fact a state variable of the system - that is, the entropy change between two specified states is the same whether the process is reversible or irreversible. Consequently, the difference $\Delta S$ between two states 1 and 2 is given by

$$
\Delta S=S_{2}-S_{1}=\int_{1}^{2}\left(\frac{d Q}{T}\right)_{\mathrm{int} \mathrm{rev}}\left(\mathrm{kJ} \mathrm{K}^{-1}\right) .
$$

In general, the change in entropy between two states is due to the entropy exchange from heat transfer and the entropy generated within the system $S_{\text {gen sys }}$ :

$$
\Delta S=S_{2}-S_{1}=\int_{\substack{1 \\ \text { Entropy transfer } \\ \text { with heat }}}^{2}\left(\frac{d Q}{T}\right)+S_{\substack{\text { Entropy generated } \\ \text { within system }}} .
$$


Following from eqn (2), it then follows that

$$
\int_{1}^{2}\left(\frac{d Q}{T}\right)+\int_{2}^{1}\left(\frac{d Q}{T}\right)_{\text {int rev }} \leq 0,
$$

that is,

$$
\int_{1}^{2}\left(\frac{d Q}{T}\right)+S_{1}-S_{2} \leq 0
$$

and from eqn (4),

$$
\Delta S \geq \int_{1}^{2}\left(\frac{d Q}{T}\right)
$$

From eqn (5) this means that

$$
S_{\text {gen sys }} \geq 0 \text {. }
$$

Thus, though the total entropy change of a given system can be negative due to the first integral involving the $(d Q / T)$ term on the right hand side of eqn (5), the entropy generation $S_{\text {gen sys }}$ cannot be negative. The entropy generation term $S_{\text {gen sys }}$ is entirely due to the irreversibilities within the system.

For an isolated or adiabatic closed system then, from eqn (6) it follows that

$$
\Delta S \geq 0
$$

There are important distinctive types of systems. An isolated system is a system where nothing at all is transferred across the boundary of such a system - that is, neither heat, work nor mass. For a closed system, only mass cannot cross the boundary, but heat and work can. In an open system mass, heat and work can all cross the boundary. The equations given earlier have been stated for a closed system. That is, the explicit possible dependence of $Q$ on mass flow has not been included. However, the principle of entropy change can be worked out for an open system as well. If an open system is being considered where mass can flow in as well, then there will be mass flow terms to include [29]. However, the statement still remains true that though the total entropy change of a given system can be negative, the entropy generation $S_{\text {gen sys }}$ cannot be negative.

The preceding discussion has been concerning the formal expression of entropy reduction that can arise locally and such reasoning has often been used for proposing an ordering principle on the basis that an entropy increase elsewhere in the Universe 'pays' for the loss term locally (see Section 2 earlier). This idea is behind the work of Martyushev and Seleznev [13] and their maximum energy production principle (MEPP). It is also behind the idea of an 'entropy flux' which Styer [30] invokes for his calculations using the statistical definition of entropy (as a microcanonical ensemble of system states analogous to the Maxwell-Boltzmann theory of heat with a probability distribution of molecular vibrations). Both Styer [30] and Bunn [31] calculate by slightly different routes a statistical upper bound on the total entropy reduction necessary to 'achieve' life on earth. This is then compared to the total entropy received by the earth from a rate of solar radiation estimated to be absorbed by the earth for a given period of time. However, all these authors are making the same assumption - viz. that all one needs is 
sufficient energy flow into a closed system (or open system, where mass flow is allowed) and this will be the means of increasing the probability of life developing in complexity and new machinery evolving. But as stated earlier this begs the question of how a local system can possibly reduce the entropy without existing machinery to do this. It is this matter which is addressed in the next section - all important is the definition of what is meant by a machine. It will be seen that crucial to this whole issue is the inability of a given system to naturally raise the free energy locally without new machinery (and thus intelligence) being involved - machines need to be pre-existing to enable an increase in order and complexity to take place.

\section{MACHINES, FREE ENERGY AND NON-ISOLATED SYSTEMS}

To make progress in understanding the concept of information and how it will impinge on the local thermodynamics anywhere in the system, it is important to carefully define our terms. A non-isolated system (either closed or open) clearly brings with it the possibility of extra energy coming into the system which may be able to do work, but will that of necessity reduce the local entropy? Will simply adding energy to a system reverse the loss of useful energy?

\subsection{Machines}

Fundamental to all useful energy flow is the concept of a machine. In a similar vein to the work of Ji [32], we make the following definition:

Definition: A machine is a functional device which uses energy.

And its corollary:

Corollary: Not everything which has a function is a machine.

As in Ji's work, one can further classify machines according to inputs, internal states and outputs, but for our purposes it is simply the use of free (available) energy which is the main distinction that is important here. The way to distinguish between a machine according to the definition we have stated and other functional objects is to consider various devices. A bottle according to this definition has function, but is not a machine, but an electric kettle is. A piston on its own clearly has function but is not a machine until connected to a cylinder and power source such as steam or the products of hydrocarbon combustion as in a car engine.

Machines are able to do work and will vary in efficiency but there will always be a loss of energy for any given working machine. This per unit of degree is the entropy of the working machine.

Consequently, another way of considering a machine is as a device for capturing energy from its surroundings such that it will be able to constrain such energy to do work.

\subsection{Non-isolated systems and Gibbs free energy}

Suppose we have a non-isolated system which has internal entropy $S_{\text {int }}$. Such a system is connected to its surroundings having entropy $S_{\mathrm{ext}}$. According to the second law for the total system, then

$$
\Delta S_{\mathrm{int}}+\Delta S_{\mathrm{ext}} \geq 0
$$


$\Delta S_{\text {ext }}$ is defined as

$$
\Delta S_{\text {ext }}=-\frac{\Delta Q}{T} \geq 0,
$$

where $\Delta Q$ is the energy gained from the surroundings, so that $-\Delta Q / T$ is entropy gained by the surroundings and

$$
\Delta S_{\text {int }}-\frac{\Delta Q}{T} \geq 0,
$$

and temperature $T$ is the same for the two systems. Multiplying both sides by $T$ we have

$$
T \Delta S_{\text {int }}-\Delta Q \geq 0 .
$$

Noting $H$ as the enthalpy of the system then $\Delta E+\Delta W=\Delta H$, where $H=E+P V$ (loosely $H$ is the 'overall thermodynamic energy available'). For a gas expansion system this leads to $\Delta H=\Delta E+P \Delta V$, and if the process is now assumed to be isobaric, then $\Delta H=\Delta Q$ so that $T \Delta S_{\text {int }}-\Delta H \geq 0$. Consequently,

$$
\Delta H-T \Delta S_{\text {int }} \leq 0,
$$

for a possible process to occur. If the Gibbs free energy is defined as

$$
G \equiv H-T S_{\text {int }},
$$

then the change $\Delta G$ in Gibbs free energy is

$$
\Delta G \equiv \Delta H-T \Delta S_{\text {int }} .
$$

Note that this is now defined entirely in terms of inner system states, and we now have a powerful tool for examining non-isolated systems. [A similar parallel approach using Helmholtz free energy $(A \equiv E-T S)$ can be taken for systems involving changes in pressure and temperature such as explosive processes, and different forms of the Helmholtz free energy find expression in other branches of physics such as electrical engineering - see, for example, Rey [34].] As a consequence of the second law of thermodynamics applied now to such nonisolated systems we can state that the following applies concerning the spontaneity of chemical reactions:

$$
\begin{array}{ll}
\Delta G<0 & \text { favoured reaction - spontaneous, } \\
\Delta G=0 & \text { reversible reaction - equilibrium, } \\
\Delta G>0 & \text { disfavoured reaction - non-spontaneous. }
\end{array}
$$

Even if material exchange was involved and one had a completely open system, no amount of matter or energy exchange without information exchange would alter the fundamental finding [eqn (16)] concerning spontaneity of chemical reactions.

More detail on how the equilibrium reaction rate constant is linked to the Gibbs free energy is given in reference [2]. All chemical reactions without external influences will minimise G. Furthermore, any natural process occurs spontaneously if and only if the associated change in Gibbs free energy $G$ for the system is negative $(\Delta G<0)$. Likewise, a system reaches equilibrium when the associated change in $G$ for the system is zero $(\Delta G=0)$, and no spontaneous process will occur if the 
change in $G$ is positive ( $\Delta G>0$ ). It is this latter case which is of greatest interest inasmuch as it connects to our earlier definition of a machine.

\subsection{Machines and free energy}

Suppose we have machine $M$ which, given an energy supply, can do work $W$. The Gibbs free energy $G_{M}$ is found by knowing the entropy $S_{M}$ of $M$ so that using eqn (14) we have in specific notation (i.e. free energy $g$, enthalpy $h$ and entropy $s$ per unit mass):

$$
g_{M} \equiv h_{M}-T s_{M}
$$

\subsubsection{Example: piston and cylinder}

\section{Piston and cylinder on its own}

Consider a simple machine, where a piston is in a cylinder. The temperature of the air in the cavity initially at atmospheric pressure (Fig. 1) is raised from $300 \mathrm{~K}$ to $400 \mathrm{~K}$ and the volume halved. Using the ideal gas law for the two states 1 and 2 we have

$$
\frac{P_{1} v_{1}}{T_{1}}=\frac{P_{2} v_{2}}{T_{2}} .
$$

Note that the first law of thermodynamics [eqn (1)] with the ideal gas law can be expressed as

$$
c_{V} \frac{d P}{P}+c_{P} \frac{d v}{v}=d s
$$

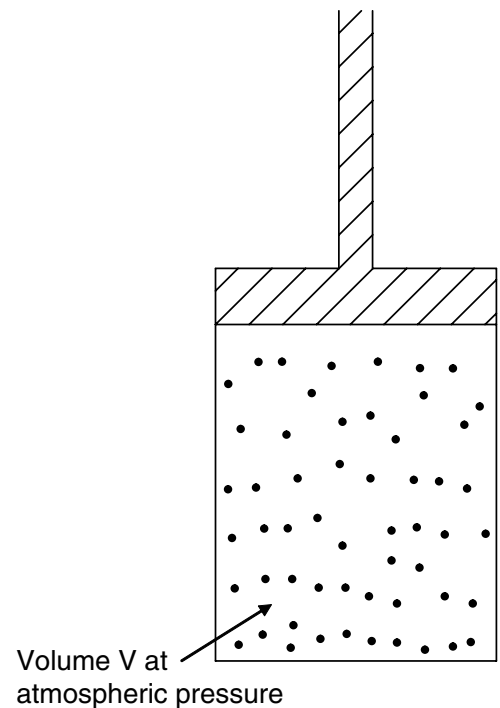

atmospheric pressure

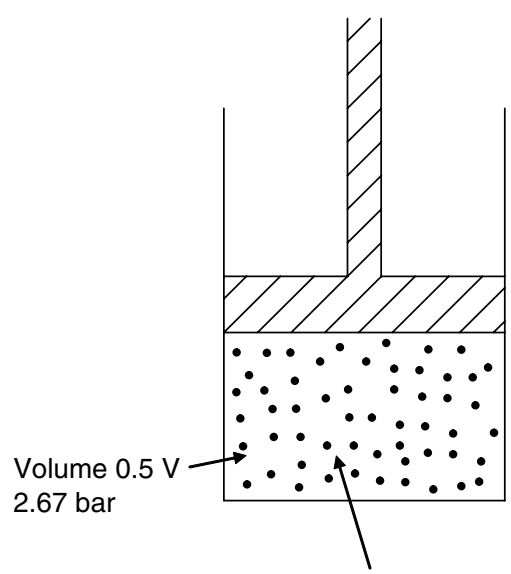

Air raised from $300 \mathrm{~K}$ to $400 \mathrm{~K}$ under isentropic compression, with no losses.

Figure 1: Piston and adiabatic (isentropic) compression. 
where $v$ and $s$ are specific volume (i.e. volume per unit mass) and specific entropy (entropy per unit mass), respectively, and $c_{v}$ and $c_{p}$ are the specific heats at constant volume and constant pressure, respectively. Integration of eqn (19) leads to the connection

$$
P v^{\gamma}=\mathrm{k} \exp \left(s^{*}\right)
$$

where $\gamma$ is the ratio of specific heats $\left(\gamma \equiv c_{P} / c_{v}\right)$ and $s^{*}$ is the non-dimensional entropy $\left(s^{*} \equiv s / c_{v}\right)$. Thus, the two states 1 and 2 for isentropic conditions obey

$$
P_{1} v_{1}^{\gamma}=P_{2} v_{2}^{\gamma} .
$$

With $v_{2}=0.5 v_{1}$, the pressure then for the second state can be shown from eqns (18) and (21) to be $P_{2}=2.67$ bar. The work done $\int_{1}^{2} P d v$ on the gas in the cylinder has led to a change in the enthalpy of the system from $h_{1}=c_{P} T_{1}$ to $h_{2}=c_{P} T_{2}$, where both $h_{1}$ and $h_{2}$ are enthalpies in specific terms (i.e. enthalpy per unit mass). The change in the free energy for the system $M$, state 2 is simply

$$
\Delta g_{M}=h_{2}-h_{1}=c_{P}\left(T_{2}-T_{1}\right)
$$

and in non-dimensional terms,

$$
\frac{\Delta g_{M}}{c_{V} T_{1}}=\gamma\left(\frac{T_{2}}{T_{1}}-1\right) .
$$

With $\gamma=1.4$ for air, and $T_{1}=300 \mathrm{~K}$ and $T_{2}=400 \mathrm{~K}$, the free energy in non-dimensional terms is 0.47 . With $c_{v}=722.9 \mathrm{~J} \mathrm{~kg}^{-1} \mathrm{~K}^{-1}$ (for air at $400 \mathrm{~K}$ ) the free energy dimensionally is $101,206 \mathrm{~J} \mathrm{~kg}^{-1}$.

Supposing we have losses in the system (Fig. 2) such that $S_{2}^{*}=0.1$ and regarding the reference state 1 as having $S_{1}^{*}=0$, then the non-isentropic state 2 is connected to state 1 through

$$
P_{1} v_{1}^{\gamma} \exp \left(-s_{1}^{*}\right)=P_{2} v_{2}^{\gamma} \exp \left(-s_{2}^{*}\right)
$$
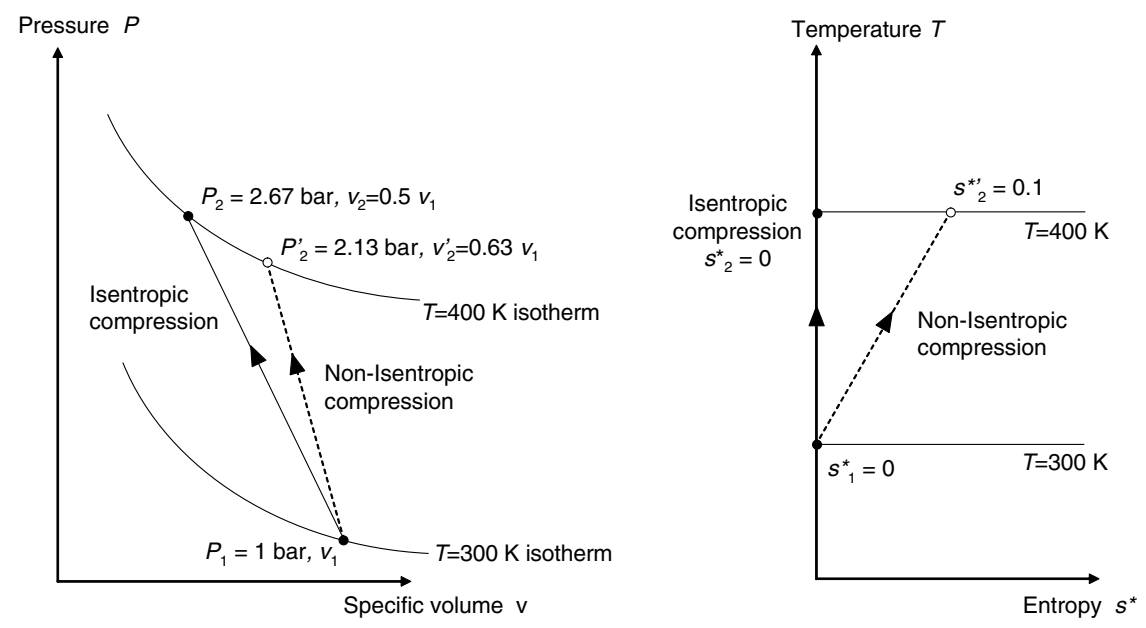

Figure 2: Pressure-specific volume; temperature-entropy diagrams for piston and adiabatic (isentropic) compression, then (dotted) near isentropic compression. 
Equations (18) and (24) with $S_{1}^{*}=0$ and $S_{2}^{*}=0.1$ lead to less compression with a new $v_{2}{ }^{\prime}=0.63 v_{1}$ and a lower pressure $P_{2}^{\prime}=2.13 \mathrm{bar}$. The change in the free energy is given by

$$
\Delta g_{M}=c_{P}\left(T_{2}-T_{1}\right)-T_{2} s_{2},
$$

and non-dimensionally,

$$
\frac{\Delta g_{M}}{c_{v} T_{1}}=\gamma\left(\frac{T_{2}}{T_{1}}-1\right)-\frac{T_{2}}{T_{1}} \frac{s_{2}}{c_{v}} .
$$

In this case,

$$
\frac{\Delta g_{M}}{c_{v} T_{1}}=0.47-0.13=0.34
$$

In dimensional terms, the drop in the free energy due to the entropy losses as the piston compresses the air is $28,916 \mathrm{~J} \mathrm{~kg}^{-1}$ and the actual free energy available is $72,290 \mathrm{~J}$. This amount of free energy is available if we allow the piston to move upwards again and the pressure to equilibrate. The change from no machine and zero free energy to a machine with $g_{M}=72,290 \mathrm{~J} \mathrm{~kg}^{-1}$ means that $\Delta G>0$; that is, this is a non-spontaneous change. With the work done on the piston it is now in a 'primed' state to do $72,290 \mathrm{~J} \mathrm{~kg}^{-1}$ of available work.

Pump used to drive the piston compressing the air

Now consider the machine used to do the work on the piston in compressing the gas. This may be a pump of some kind (it could be electrical or water- or hand-operated). The calculations are in specific terms - that is, in terms of per unit kilogram of air compressed in the piston system. Suppose the amount of energy used by the pump is much greater than $72,290 \mathrm{~J} \mathrm{~kg}^{-1}$ because of the inefficiency of the pump, frictional bearing heat losses, etc.; say this is $100,000 \mathrm{~J} \mathrm{~kg}^{-1}$. That is, there is a wastage of $27,710 \mathrm{~J} \mathrm{~kg}^{-1}(100,000-72,290)$. With a working temperature of the pump of $300 \mathrm{~K}$ this would represent an entropy of $92.37 \mathrm{~J} \mathrm{~kg}^{-1} \mathrm{~K}^{-1}$. Then with the pump being applied, the energy balance for the pump, as it works on the piston and cylinder, is:

$$
g_{P} \equiv-100,000+27,710=-72,290 \mathrm{~J} \mathrm{~kg}^{-1} .
$$

That is, for every kilogram of air compressed in the piston system, from the perspective of the pump system alone, there is a loss of free energy $\left(100,000 \mathrm{~J} \mathrm{~kg}^{-1}\right)$ which goes into $27,710 \mathrm{~J} \mathrm{~kg}^{-1}$ wasted energy and $72,290 \mathrm{~J} \mathrm{~kg}^{-1}$ to give free energy to the piston compressing the air. Once the pump power is engaged, notice that $\Delta g_{p}<0$. That is, this is a spontaneous change. There is nothing stopping it. There is energy available from the necessary machine (the pump), so the transfer of free energy to the piston is successful in priming the piston to a raised free energy.

Solar cells used to move the piston

Now consider the machine used to move the piston to be an array of solar cells (connected to an electric pump to drive the piston). Again the calculations are in specific terms - that is, per unit kilogram of air compressed in the piston system. Suppose that this time there is a trigger level of power such that energy is not conveyed to bring power to the piston, unless that power (energy per unit time) is met. Much energy is not used in this case because the trigger is not met sufficiently. It might be, say, 200,000 J kg-1 that is expended in solar energy falling on the array of solar cells before the pump is finally activated. This time it is $200,000-72,290=127,710 \mathrm{~J} \mathrm{~kg}^{-1}$ wasted, which, for a 
temperature of $300 \mathrm{~K}$, is equivalent to an entropy of $425.7 \mathrm{~J} \mathrm{~kg}^{-1} \mathrm{~K}^{-1}$. Then, the energy balance for the array of solar cells as it works on the piston and cylinder is:

$$
g_{\text {solar }} \equiv-200,000+127,710=-72,290 \mathrm{~J} \mathrm{~kg}^{-1} \text {. }
$$

That is, for every kilogram of air compressed in the piston system, from the perspective of the solar cell system, there is a loss of free energy $\left(200,000 \mathrm{~J} \mathrm{~kg}^{-1}\right)$ which goes into $127,710 \mathrm{~J} \mathrm{~kg}^{-1}$ wasted energy and $72,290 \mathrm{~J} \mathrm{~kg}^{-1}$ to give free energy to the piston compressing the air. Again $\Delta g_{\text {solar }}<0$ for this system and it is a spontaneous change, but with a large amount of wasted energy involved. Nothing has stopped it working, but what was the reason there was success? The reason was not due to the amount of energy available. The reason was simply that there was a pre-existing machine to transfer energy to give free energy held by the piston and cylinder system.

There is therefore an important deduction concerning machines (according to our earlier definition) and positive free energy devices.

Deduction: A positive free energy device cannot arise spontaneously. It always requires another operational machine to enable the free energy to be loaded/'primed', ready to do work.

\subsubsection{Other examples including polymers in living systems}

We give some further examples which convey the importance of stored free energy in real systems.

\section{Compressed spring}

The energy of compression of a spring (Fig. 3) according to Hooke's Law, will be approximately proportional to the shortening from its normal length. The free energy will be the energy of compression minus the small losses in the spring; to load the free energy into such a system will require the use of energy from a supply using an existing machine.

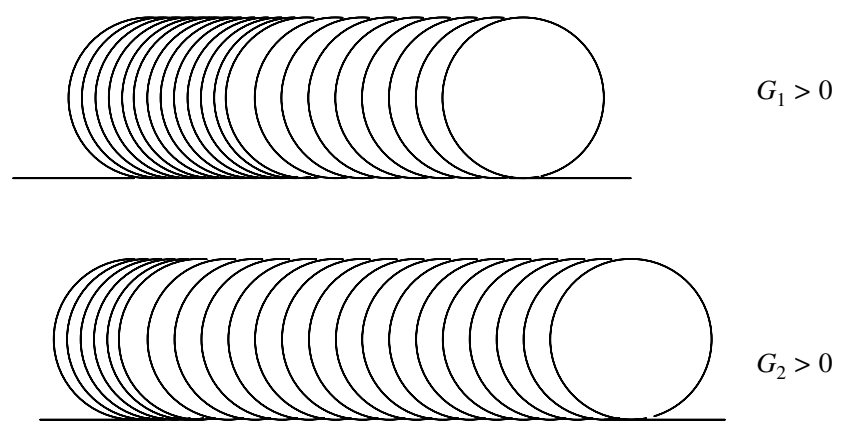

Figure 3: All natural molecule formations are like coiled springs such that if one lets the system ' $g o$ ' they 'relax': $\Delta G<0$ (due to $G \equiv H-T S>0$ ). To 'set' such springs requires an initial input of ordered energy by another machine. In this example, the internal energy in the second case $G_{2}$ is less than that of the first case $G_{1}$.

\section{DNA molecule}

Figure 4 shows a typical strand of DNA where nucleotide pairs guanine $(\mathrm{G})$-cytosine $(\mathrm{C})$ and adenine (A)-thymine (T) are positive free energy bonds across the sugar phosphate paired strands, such that left to themselves they will decay. The Gibbs free energy of such bonds is again positive $(\Delta G>0)$ so that they cannot happen spontaneously.

The best analogy is the set of compressed springs as in the previous example (Fig. 3). Furthermore, the assembly of these requires a machine which of course is the DNA polymerase protein. 


$\begin{array}{ll}\text { Main strand } & \text { ATGATTGACATTGAGGATCCAT } \\ \text { Complementary strand } & \text { TACTAACTGTAACT CCTAGGTA }\end{array}$

Figure 4: Sample genetic code with complementary strands.

This is a three-dimensional protein which places together the sugar phosphates and nucleotide bonds of the DNA molecule. Energy is supplied from the respiration of the biological phenotype feeding energy through the adenosine triphosphate (ATP) chemical motors. The same principle applies - the molecule cannot form spontaneously with only unconstrained energy. It requires a controlling machine (DNA polymerase) in order to transfer the appropriate free energy $(\Delta G>0)$ to the nucleotide bonds so that the rungs of the ladder structure of the double helix DNA form correctly [35].

It may be argued that this set of machinery could come about spontaneously but the laws of thermodynamics are completely against this. In our spring analogy, it would be equivalent to proposing that the energy that is brought in randomly somehow manages to form a set of coiled springs and maintain them in that state, such that they all keep their set energy levels. Such a notion is scientifically impossible.

The same situation in principle applies for the formation of proteins made from amino acids. The machine which does this is a ribosome, and vitally necessary before the proteins can be formed. Such polypeptide formation involves a series (usually thousands) of positive free energy bonds. In fact $\Delta H$ in the polymerisation of important organic molecules is generally always positive (20-30 kJ mol$\left.{ }^{-1}\right)$ indicating such reactions can never spontaneously occur at or near equilibrium. Even further away in disequilibrium this could not happen spontaneously unless there is an existing prior machine controlling it. Thaxton et al. [36] show that both the enthalpic and entropic (thermal and configurational) contributions to the free energy make the polymerisation process so hugely 'uphill' that the yield will be effectively zero for even simple polypeptides. As long as the laws of thermodynamics are operative, not even one biopolymer will form spontaneously without a pre-existing machine. In section 5.2.2 later we also note the extraordinary repairing ability of living systems to mend damaged DNA.

Wicken argues for 'charging' of the pre-biosphere [37] as being a means of obtaining the information to form the first living system, but the problem with this is that one really has to argue for the recharging of such a system at every step since at each stage one must have raised free energy devices to keep the whole system moving inexorably thermodynamically uphill towards the higher creatures. This is not convincing since thermodynamically at every stage the system is seeking equilibrium in the opposite direction. The Thaxton et al. [36] calculation mentioned earlier is stacked against such a process at every step.

Some have argued that beyond the first life, one could have processes whereby new machinery could arise by natural selection operating on mutations. But there are major obstacles to this view. Let us take a simple example. Suppose one starts with a specific protein - an enzyme whose purpose is to extract glucose. A series of mutations can lead to the loss of this function and in some cases the recognition of lactose as against glucose [38]. Some might argue that this is an example of the synthesis of a new machine. But this is not the case. The essentially sensory function is unaltered. It is simply the object of the function which has changed such that glucose is no longer programmed for in the DNA template that has been mutated. One has not produced a completely new machine but simply corrupted an existing one. Such conditions are not adding more than that which was there already, since they represent sub-machinery of an existing working machine. Many proteins are highly three-dimensional and their shape is crucial. Mutations always corrupt this function. One will never get a completely new configuration of folding proteins with entirely new machinery from mutationary pathways. If the argument is made that the 'new' machine could be coded for already according to a design template in the DNA, then that means that the new 
machine is, in effect, already present in embryonic form. Consequently, it is not then an example of a new machine in the definition that we have employed here. In our definition of a 'new machine', it means a machine which has not existed before and for which a DNA template of the appropriate nucleotide sequence does not exist already.

\section{Crystal formation}

Consider eqn (15) given earlier. If $\Delta H$ is negative but $\Delta S$ is also negative, then one can get cases where the net change in Gibbs free energy $\Delta G$ is zero. These cases (as referred to in Section 4.2) are examples of reversible reactions, and particularly happen at conditions of change of phase such as water going to ice crystals at $0^{\circ} \mathrm{C}(273 \mathrm{~K})$ (Fig. 5). The entropy reduction multiplied by the temperature exactly balances the drop in the enthalpy. That is in the case of crystal formation

$$
\Delta H=T \Delta S .
$$

One can liken the $\Delta S$ in this equation to being the logical/geometric influence on the thermodynamics such that the order inherent in the molecules themselves, given a low enough temperature, will cause the crystals of a particular shape to form. When such a compound is cooled to produce crystals, it is worth noting, however, that it is not the cooling itself which causes the crystals to occur, but the response to the molecular bonding which is very precise within the material and has a definite function of the state variables. Often this is regarded as demonstrating increase in order (and thus a lame argument for moving to functional form within a system) when in fact the

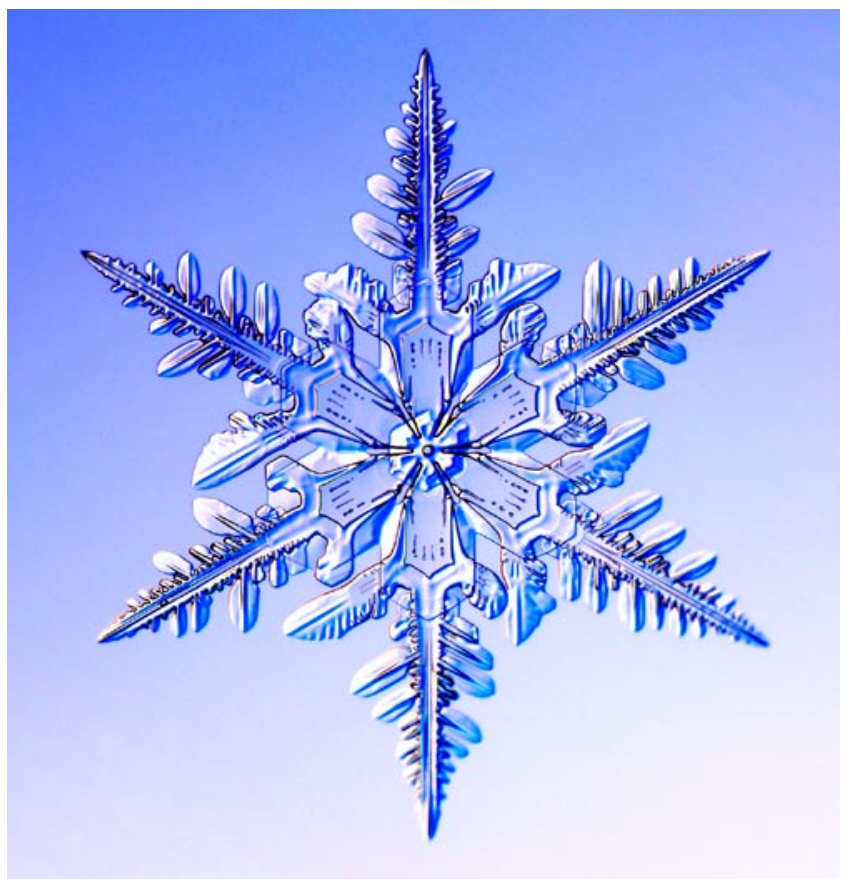

Figure 5: A snowflake is an example where entropy is lowered as the phase change temperature is crossed, but the overall Gibbs free energy change is zero. The entropy reduction is simply a reflection of the geometry and the energy bonds already existing in the hydrogen and oxygen atoms. The entropy reduction does not produce new order that was not latently there already. (With grateful permission of the owners of web site www.snowflake.com where many excellent photos of snow crystals are available). 
ordering principle is latently already there in the structure of the chemical elements to begin with. As we shall see in the next section, with the ordering principle already present, no new order is gained - order is always linked to a pre-existing machine.

In all these examples of non-isolated systems, we have mainly been concerned with closed systems (that is, only open to energy exchange, not material exchange). This clearly becomes pertinent when one considers the origin of information in the next section, where some have argued for material exchange to include transfer of configurational information (and thus lowering the entropy locally).

\section{THE VITAL ROLE OF INFORMATION}

We now address the role of information. There are three different approaches to the role of information. The first sees information as entirely materialistic - a very prevalent view today. In this view, there is no attempt to regard information in a system as being defined differently to matter and energy. Dawkins [7] and many others support this point of view. Though they accept the role of thermodynamics as basic to all matter and energy processes, they see no role for non-material information in living systems beyond the Shannon concept of information transfer, and increasing the probability of new mutational structures. The DNA code is still regarded as only an entity defined by matter and energy in this view. The second approach regards information as being akin to a nonmaterial structure and as a system input. Entropy decreases are gained locally at the expense of an overall entropy increase in the larger system behaviour. Information is fed into the system as a bottom-up concept where auto-organisation, it is argued, occurs, given the right circumstances. The third option regards information as existing and overlaying the thermodynamic structure. The fundamental approach is top-down - local machinery is constrained by coded information.

\subsection{Information - 'matter and energy is all there is . .'? the materialistic view of information}

The materialist view of the origin of life, whilst not held by all within evolutionary science, has become a dominant force in biology over the last century. The work of many authors such as Ernst Mayr, What Evolution is [7], and Richard Leakey and Roger Lewin, Origins [39], and the popularisation of this approach by David Attenborough's crafted media presentations (such as The Living Planet [40]) set the scene for what is essentially a reductionist and entirely natural approach to the origin of life. Consistent with this thinking is the idea of non-material information as an illusion and not fundamentally controlling the origin of biological function. Dawkins espouses this view [15], and follows the work of Cairns-Smith [41] who famously referred to a 'frozen accident' for the origin of the DNA code, and, in particular, the way the DNA code is both recognised by sender (DNA) and receiver (messenger mRNA/ribosome).

\subsubsection{Some remarks on self-organising systems and protein folding}

Before discussing the materialistic view of genetic information, a remark concerning self-organising systems is perhaps appropriate here.

It is well known that with a minimum amount of genetic input, some chemical systems can be regarded as self-organising. Ball in his book The Self-made Tapestry [9] illustrates this principle at work in natural systems from the self similar shape and growth of the seashell spiral (such as the nautilus) and the patterns of seeds in the head of a sunflower, to the spots of a leopard and the pelt pattern of a giraffe. Ball investigates specific patterns in depth, revealing that these designs are selforganised and that simple, local interactions between component parts produce motifs like spots, stripes, branches and honeycombs. Murray and coworkers [18, 42, 43] have composed a mathematical basis for the formation of patterns in living systems using the analysis of these structures. However, this aspect of self-organising is always reflecting a basic amount of information which is inherent in the organism. Hence, it is not self-organising in the absolute sense, that is it does not 
happen without any principle at all of organising being there to begin with. In a similar way, Maynard Smith and Szathmary [8] pick up this theme in discussing the folding of proteins once they have formed initially as a string of amino acids coded for by the genes in the DNA. In their discussion of pattern formation they draw an analogy with a picture made by an ink-jet printer [44].

Just as one thinks that biological form is stamped out, so no one thinks that biological form is made as a computer image is made. What is true, however, is that a protein molecule is made in a way analogous to such an image. Thus, there is a one-to-one correspondence between amino acids in the protein and base triplets in the gene that coded for it. Change one base and you will change one amino acid. This is not the whole story: the gene specifies the sequence of bases in the protein, but the string must then fold up to produce the three-dimensional form. In most cases, the string will fold up on its own: folding is a self-organised dynamic process, depending on the laws of physics, which do not need to be programmed.

Maynard Smith and Szathmary here rightly point out that the folding of proteins is selforganised - it is obeying the laws of physics which will ensure a particular folding pattern. However the self-organising principle is already within the system. In the book The Ten Most Wanted Solutions in Protein Bioinformatics [45] by Anna Tramontano, chapter 4 specifically deals with the problem of protein structure prediction. This work very carefully shows that the shape of proteins, though following the laws of physics (Maynard Smith and Szathmary), is in fact a function of a free energy path, which is still not fully understood, and yet 'the biochemical function of a protein is determined largely by its three-dimensional structure' [46]. Thus, it is evident that the very folding path of the proteins demonstrates in reality another vital piece of intelligent machinery which has itself been pre-programmed - thus what this exposes is that there is a deeper level of information carrying ability within the DNA-RNA-Ribosome-protein communication system which is a layer beneath the already immensely sophisticated coding part of the DNA. Thus, it was not surprising that in a report [47] in Nature of the ENCODE pilot project on the human genome by the EMBL-European Bioinformatics Institute at the Wellcome Trust in Cambridge, it was admitted that the information contained in the genetic communication system is not limited to the coding part of the DNA.

The human genome is an elegant but cryptic store of information. The roughly three billion bases encode, either directly or indirectly, the instructions for synthesising nearly all the molecules that form each human cell, tissue and organ. Sequencing the human genome provided highly accurate DNA sequences for each of the 24 chromosomes. However, at present, we have an incomplete understanding of the protein-coding portions of the genome, and markedly less understanding of both non-protein-coding transcripts and genomic elements that temporally and spatially regulate gene expression. To understand the human genome, and by extension the biological processes it orchestrates and the ways in which its defects can give rise to disease, we need a more transparent view of the information it encodes. [47]

The implication is that that which was previously thought to be junk DNA (and vestiges of ancestral connections with pre human ancestors) may in fact be carrying vital information after all, the 'language' of which has yet to be discovered.

5.1.2 Reductionist view of genetic information in the coding part of DNA

Held by the majority of the scientific community, the reductionist approach regards the coding and language of DNA as essentially a phenomenon of the physics and chemistry of 
the nucleotides themselves - this and the associated coding of ordered amino acids to make proteins.

The emergence of nucleotide binding sites, that is, locations on the DNA or RNA which serve as addresses made of the sequence of bases Adenine A, Cytosine (C), Guanine (G), Thymine (T)/Uracil (U), which specialised proteins recognize, and can identify and bind to, is vital to theories of the evolution of biological information such as those promoted vigorously by Schneider [48]. He attempts to simulate the emergence of genetic binding sites numerically and claims that these regulatory sequences could increase information through evolutionary processes starting from a random DNA sequence. The programming of Schneider assumes that there are miniscule organisms, each with full transcription and translation machinery, and that these organisms are able at random to site nucleotides at particular sites. However, there are some important assumptions in his work, particularly a very high (and unrealistic) mutation rate as well as a totally arbitrary and high number of these mutations assumed to be instant providers of reliable binding interactions. Truman [49] has thoroughly refuted Schneider's false claims and shown that the program he has written is far from a simulation of reality. As he rightly states [50]:

One could write a computer program which 'shows' that random natural processes would drive rocks from a quarry up a steep mountain in thousands of discrete steps, for every simulation run. One only has to use an unrealistic number of earthquakes and improperly model the effects not leading to our intended programming goal. The details matter very much to determine the true net outcome... . Overlooked details in such flawed simulations might not be obvious. Vast numbers of unrealistically hard earthquakes would affect not only the movement of our rocks but the surrounding mountain would be systematically destroyed.

A legitimate simulation must reflect what is being modelled with sufficient accuracy to justify decisions ... The true expected net outcome depends on the details.

A yet more serious challenge comes earlier from the writings of the eminent author Kimura who in a series of much quoted papers [51-56] has argued that natural selection is the process of accumulating genetic information in adaptive evolution. But from Kimura's own work it is evident that there is a whole raft of deleterious genetic mutations which are effectively neutral in terms of phenotypic effect. The Kimura distribution of frequency of mutation occurrence against its effect [57] is now a standard and well-established finding for biological systems although rarely plotted out. In particular, the peak of the mutation rate is actually in a region of neutral effect with little, if any, beneficial mutations observed in practice. Sanford [58] shows rightly that the curve is not at all bellshaped, but is highly skewed away from a normal distribution. The important conclusion is that the vast majority of near-neutral mutations are deleterious and have no effect on the organism, and there are some beyond the neutral region which cause serious genetic disease. Sanford has shown that in reality the very small number of beneficial mutations is far outweighed by the deleterious, and that this all occurs in the neutral region so that they are not felt anyway at the phenotypic level. The work of Kim et al. [59] (and other authors in a similar approach) seeks to measure the increase of entropy $H=-\Sigma_{1} p_{i} \ln _{2} p_{i}$ of a signal, where $p_{i}$ is the probability of the occurrence of state $i$. The aim is to provide a means of increasing the genetic possibilities (changes) within the phenotype by a vast build up of such mutations (see Dawkins [15]) and then appeal to natural selection to weed out the changes which are truly of benefit to the organism. Most are not functional (and in fact lethal) butsome are functional and survive. However, in practice the work of Kim and other authors seeking to determine systematically the Shannon entropy (information) increase from genetic mutations is in fact largely not relevant to functional information at the phenotype level for the following reasons: 
1. Such mutational changes are largely within the neutral zone of the Kimura distribution and therefore not felt at all at the phenotype level.

2. Shannon entropy is not in fact the true metric for measuring increase in phenotypic possibilities (this is discussed in Section 5.3 below). In many ways Shannon entropy is the opposite of what is really needed, since it is really a measure of the spread of mutations at the nucleotide level, and these mutations are invariably deleterious (from the discussion above concerning the Kimura distribution).

3. Even if one does insist on equating Shannon information with phenotype changes chosen by natural selection, Kimura [60] and indeed (much earlier) Haldane [61] both agree it is not possible to select for a large number of traits simultaneously due to the immense genetic cost at the phenotype level of such selective pressures [62].

5.1.3 True biological information and the materialist cul-de-sac

In summary, Maynard Smith typifies the distinctly reductionist viewpoint when he states [63]:

Information enters biology when there is a distinction between genotype and phenotype. When did this arise? It is now widely agreed that the present DNA-protein world was preceded by an 'RNA world' [64], in which the same RNA molecules were both replicator and enzyme. Such entities would meet Muller's [65] definition of living: they possessed multiplication, variation and heredity, and hence were capable of adaptive evolution. But there was no genetic code one cannot code for oneself - and hence no transmission of information.

And therein lies the immense difficulty with this approach. Forced by reductionist thinking, Maynard Smith proceeds in the same article to suggest that it may even not have been necessary for DNA coding to exist before the emergence of life. But the key is his statement 'one cannot code for oneself'. If there is an a priori assumption of materialism, then one is forced into such thinking.

To consider biological information as simply a 'by product' of natural selective forces operating on random mutations is not only counter-intuitive, but scientifically wrong for the following three reasons:

Firstly, the irreducibly complex nature of the machinery involved in creating the DNA/mRNA/ ribosome/amino acid/protein/DNA-polymerase connections (apart from the energy supply needed from the ATP motor). All of these functioning parts are needed to make the basic forms of living cells to work. These arguments are well rehearsed elsewhere by Trevors and Abel [66] but are formidable and essentially unanswered. This, it may be argued, is a repeat of the irreducible complexity argument of Behe [67], and many think that that debate has been settled by the work of Pallen and Matzke [68] where an attempt to explain the origin of the bacterial flagellum rotary motor as a development of the Type 3 secretory system has been made. However, this argument is not robust simply because it is evident that there are features of both mechanisms which are clearly not within the genetic framework of the other. That is, the evidence, far from pointing to one being the ancestor of the other, actually points to them both being irreducibly complex. In the view of the author this argument is still a very powerful one.

Secondly (as detailed in Section 4.3), at a material level the machinery involved requires the setting of free energy levels in banks of molecular structures, all of which have to be at their right level for a protein or a DNA string of nucleotides to operate. It is against the known principles of thermodynamics in physics and chemistry for this to happen spontaneously.

Thirdly, the encoded information is not inherent in the physics and chemistry itself. There is no chemical reason for the $\mathrm{G}-\mathrm{C}$ or A-T bond to form and make the rungs of the ladder of DNA (indeed these do not form spontaneously). Furthermore, the order of the nucleotides in a string of coding is not predetermined by the chemistry. The evidence itself strongly argues against the materialistic position. 


\section{2 'Bottom-up' approach: information is non-material but has arisen by} low entropy systems maximising the entropy in their environment

With some variations between them, this is the view held by Prigogine [3-6], Wicken [10], Yockey [69], Demetrius [70] and Kauffman [11]. Essentially, they all recognise that in some way information is indeed non-material, and that there is a transfer of 'logical entropy' locally to produce order and structure in a biological system. They take the approach that information is gained by the 'entropy' of the signal (the Shannon concept discussed earlier in the introduction). This increases the variations introduced into the code carrying DNA and thus feeds 'possibilities' for natural selection to operate on. It is then maintained that a lowering of the local entropy is achieved by such natural selective processes giving advantage at the phenotype level. Local complexity is gained at the expense of increased disorder elsewhere (the maximum entropy principle [13]). This theory has been developed using nonequilibrium thermodynamics, particularly by the authors Wiley and Brooks $[12,71]$ who whilst claiming that evolutionary development is fed by low entropy, do recognise that not all can be explained this way. A frank admission occurs in a dialogue with Wicken of the authors Brooks and Wiley [72]:

Ecological influences acting upon truly random variation could not produce the biological organisation we see today. Something else must be working as well. To us, that 'something else' is the self-organising abilities of living things stemming from their non-equilibrium nature and the fact that their histories, embodied in the genes and cytoplasmic organisation they receive from their parents, form the initial conditions from which they develop.

As argued in section 5.1.1 the appeal to self organization only shifts the problem to a different arena since any such self organizing systems would only reflect sophisticated chemical machinery already present, and section 4.3.2 has already demonstrated that the DNA bonds and protein bonds do not happen spontaneously. Indeed Paul Davies [73] makes the point that the coding of DNA shows that

"Life is not an example of self-organisation. Life is in fact specified...living things are instructed by the genetic software encoded in their DNA (or RNA)".

\subsubsection{Autocatalytic reactions and origin of life studies}

Kauffman [11] along with Wicken [10] argues for the rise of low entropy information systems by use of autocatalytic chemistry. Their primary concern is to propose a logical pathway whereby polymers needed for living systems could emerge. Their studies are really concerning the study of the issue of the origin of life - abiogenesis.

Wicken in his book Evolution, Thermodynamics and Information [10] treats the earth and its atmosphere as a thermodynamically closed system and argues that in a pre-biotic soup, the energy from the sun will cause information to increase [74] by causing the Gibbs (canonical) ensemble (which represents a probability distribution of all the microscopic states of the system) of free energies to increase. $\mathrm{He}$ argues that the information increase is maintained by free energies rising in the microstates of the system whilst at the same time there being an increase in the number of different molecular configurations, and so overall having an increase in the entropy of the total system. Basic to the thesis of both Wicken and Kauffman and all this school of thinking (Demetrius, Yockey, Prigogine, Wiley and Brook) is the concept that the second law of thermodynamics in their thinking provides a means of generating variety and enabling natural selection to tune such variety to conservable phenotypes and thus provide fitness to survive. Thus, biological information is thought of as a product of the randomisation of microsystems (or mutations at the genetic level). Though Wicken is not entirely at ease with this concept (as we now discuss), we note this common theme from 'bottom-up' theorists and later in Section 5.2.2 we 
suggest that this is far from the reality of what functional information in biological systems really entails. In Section 5.3, we suggest a much more viable alternative.

Wicken refers to 'microspheres' as dynamic entities, capable of growth and reproduction [75]. In Wicken's thinking information 'evolves only within a context of utilisation' [76]. And for Wicken this is where there are particular difficulties with his work; since he is primarily dealing with the small scale thermal/chemical entropies and using the tools he has, he is unable to really address the macroscale as he has no informational hierarchy in his proposition. As Collier [77] has pointed out,

'Functional organisation at the organic level cannot be explained in terms of energetics alone, since the self-organising processes governing the formation of gross phenotypic characters are not obviously energetically driven'.

But though he may fail to convincingly make the connection from the chemical/thermal to the concept of the large scale functional information at the phenotype level, what Wicken does show in his important attempt to bridge this gap is that there really is an important conceptual difference between chemical and informational processes. Indeed Wicken denies any meaning to the formal isomorphism that others attempt between entropy and information-carrying capacity (as we noted earlier) on the grounds that there is no known scientifically testable necessity for that capacity to increase. In this, Wicken is correct. Whilst others persist in trying to connect the two, Wicken in fact has indicated the seed of the answer which we suggest in Section 5.3 that information sits as a nonmaterial entity on a material free energy system.

Kauffman in his work takes fundamentally the same bottom-up approach but assumes far from equilibrium thermodynamics and proposes a means of connecting the chemical/thermal entropy and information through systems of autocatalytic entities which feed negative entropy and thus free energy into the building blocks of life. Kauffman [78] states,

I believe it [life] to be an expected, emergent, collective property of complex systems of polymer catalysts. Life, I suggest, 'crystallises' in a phase transition leading to connected sequences of biochemical transformations by which polymers and simpler building blocks mutually catalyse their reproduction.

However, despite Kauffman's optimism, the thermodynamic obstacles are severe. He acknowledges this [79] and takes an example of forming a peptide bond in a high water concentration of 55 $\mathrm{M}$ (i.e. molarity - moles of solute/litres of solution). In water, the peptide monomer can form spontaneously $(\Delta G<0)$ but the thermodynamics is strongly against polymer formation. Thus, the stringing together of many attached monomers does not happen in practice. He correctly states that 'the concentration of large polymers at equilibrium decreases exponentially with length'. That is, the likelihood of finding large polymers such as are needed with proteins is small. A rather plaintive statement concludes this initial thermodynamic discussion - 'organisms must use complex strategies to circumvent this fundamental difficulty'.

Undaunted, Kauffman then proceeds to argue that autocatalytic reactions are the answer, since they can cause a much greater occurrence of polymers in an unsteady but slowly changing environment. He poses a thermodynamically open system where existing monomers and small polymers are allowed to be introduced to the incubator pool and treats this as an open dynamical system (called an excitable system in non-linear dynamical theory). Certainly there is evidence that autocatalysis does indeed increase the temporary emergence of longer polymers. Sustained concentrations of small and large polymers can exist if the dynamical system receives sufficient drip feed of additional monomers - a critical point then is reached which causes the excitable system to move into a slow sustained limit cycle around a new attractor in parameter space. 
However, this scenario is in reality a long way off the necessary thermodynamic linkages required in polymers in RNA, DNA and proteins. In his simulations Kauffman [80] tuned the dehydration of the system to favour the synthesis of peptide bonds, and assumed an idealised scenario where the rate of catalysed reactions is much faster than the rate of spontaneous reactions. There are three major arguments against the autocatalytic thesis of Kauffman and Wicken, and though more sophisticated, the theories of Wiley and Brooks:

1. Firstly, the sustained existence of polymers without the high concentration of water is still not possible by the ingenious limit cycle proposition. As soon as the influx of polymers is removed, or simply reduced, the probability of long polymers being sustained is lowered to negligible levels. As explained earlier in Section 4.3.2 the polypeptide formation in real proteins requires thousands of carefully ordered positive free energy bonds. The arguments of Thaxton et al. [36] are essentially unmatched by Kauffman's thesis precisely because he is not really addressing the final end game, which still requires in essence a monumental series of 'uphill' processes to line up precise values of positive free energy steps in the peptide bonds.

2. The second major difficulty is the homochirality issue. When Harold Urey and Stanley Miller [81] in 1953 managed to produce amino acids by passing an electric charge through a reducing atmosphere of methane, ammonia, water and hydrogen, they did produce amino acids with some (but by no means all) of the standard 20 amino acids which need to be coded for in order to make the proteins for life. But the most important difficulty was that they produced both left handed and right handed chirality molecules in the expected 50:50 ratio. However, living systems have entirely left handed versions of these molecules (i.e. homochirality) which otherwise are identical in chemical behaviour. Living systems are not just to do with chemicals but with the shape and positioning of the chemicals. Miller acknowledged that the difficulties were indeed formidable when in 1991 he stated to Scientific American [82] that 'the problem of the origin of life has turned out to be much more difficult than I, and most other people, imagined ....

3. The third major difficulty that Kauffman's thesis does not adequately address is the problem of the information in the molecules of DNA and RNA which code for the appropriate peptide bonds and form the string of amino acids. He resorts to the idea of a 'random grammar' in chapter 10 of his book [83]. Such ideas are rooted in the notion that a grammar of communication emerges once one has achieved a set of autocatalytically connected set of symbol strings. The hard evidence for this is in fact not experimentally available and it is only on the basis of an initial recognition of functional coupling that any numerical predictive programs [84] can be made to produce a simulated 'grammar' to begin with between DNA and proteins. The experimental verification of such a thesis is blocked precisely because of the thermodynamic hurdles of (1) and (2) in this list. Kauffman's aims are optimistic because he has by his own admission insisted on a set of extremely tenuous starting conditions [85]

It behoves our caution when thinking that all transformations might take place under common conditions abiogenetically. If not, then the congregation of these precursor compounds may be problematical. Be that as it may. Let us accept the sympathetic view: Let there be an abundance of small organic molecules in the promised dilute, early ocean, tidal pool, geyser basin, atmospheric droplet. This not the problem I want to attack.

The problem I do want to attack is this: How hard is it to obtain self-producing systems of complex organic molecules capable of a metabolism coordinating the flow of small molecules and energy needed for reproduction and also capable of further evolution? Contrary to our expectations, the answer, I think, is that it may be surprisingly easy. 
For the reasons stated earlier, Kauffman is trying to push against the obvious downhill tendency of the thermodynamics at every stage and appealing to a 'sympathetic view', when in fact the very problem he does not wish to attack is the key to unlocking the problem he does wish to attack. That is, organic molecules (small or otherwise) cannot form spontaneously if the machinery/information is not already present to do so. There is a straightforward alternative of a top-down approach to the information carrying molecules of life. This is developed further in Section 5.3 below.

In this bottom-up approach, Kauffman, Wicken and others are attempting to find a thermodynamic justification for their belief in information generation by evolutionary processes by searching for answers beyond the purely neo-Darwinian synthesis [86]. They fundamentally believe that it must be so, but are really no further than the optimism expressed by Wicken [87] in 1979.

Evolutionary processes are anamorphic, or complexity-generating. The passage of evolutionary time is accompanied by the emergence of structures having progressively greater morphological and functional complexity. But the essential nature of evolutionary anamorphosis remains enigmatic. It has not been successfully derived from or identified with more fundamental physicochemical laws, particularly those of thermodynamics; nor has it been adequately explained at its own phenomenological level by current evolutionary theory. Neo-Darwinism in particular seems to have enormous difficulty in accounting for this fundamental feature of evolutionary process.

It should be acknowledged that Wicken in Evolution, Thermodynamics and Information [10] and Kauffman in Origins of Life [11] are expressing their confidence in abiogenesis (referred to as Class 2 biological systems) rather than the day-to-day operation of biological systems (Class 1 systems). However, even the development of advanced molecular machinery once one has 'simple' replicating DNA or RNA structures (that is, Class 1 systems after abiogenesis) is still up against formidable obstacles. The importance of sequence complexity and its relevance to biopolymeric information is discussed in the papers by Abel and Trevors [88, 89]. This represents a major hurdle to build a software messaging system. However, even before that, there are major thermodynamic hurdles as explained in the following Section 5.2.2.

\subsubsection{Logical entropy transfer}

The work of Prigogine and others [3-6] has formalised the thesis of the bottom-up approach and greater detail can be found on this point in the earlier conference paper [2] of the author. The main arguments of this article are based on the data presented in that conference paper. Prigogine proposed the addition of other entropies which could feed negative entropy into a given thermodynamically closed or open (mass exchanging) system. In his writings the total entropy is considered to be

$$
d S=d S_{T}+d S_{\text {logical }},
$$

where $d S$ is the total change in entropy, $d S_{T}$ is the change in thermodynamic entropy and $d S_{\text {logical }}$ is the change in entropy due to complexity - that is, 'Prigogine entropy' or 'logical entropy'. For any system with an exchange of energy there will be an appropriate way of describing both the thermodynamic internal energy (essentially thermal), the work done (electromagnetic, thermal, kinetic, etc.) and the entropy $d S_{T}$ (as discussed earlier in Section 4). While $d S_{T}$ tends to increase, the term $d S_{\text {logical }}$ can increase or decrease or remain zero (it is considered positive if entropy enters the system and negative if entropy leaves the system). The important implication of the additional logical entropy term is that then the total entropy change of any open system, $d S$, can 
be considered positive, negative or zero. Systems for which $d S<0$ (that is, where entropy is decreasing) are said to be self-organising (Cambel [90]), though this term needs care since the organising is only reflecting an ordering principle already present. The example of crystallisation given earlier (Section 4.3.2) is where $d S_{\text {logical }}$ represents the order inherent in crystals due to the atomic structure of a particular chemical compound. And as we have already indicated, it is not the cooling itself which causes the crystals to occur, but the response to the precise molecular bonding within that compound which gives the lowering of entropy, and the change in free energy is precisely zero.

According to this view of logical entropy the increased variation singled out by natural selection leads to reduced logical entropy locally. There are some very laudable attempts to bring together a case for moving from molecular order to working functional systems in biology. The work of Goonatilake [16] acknowledges the non-material nature of information but appeals to a bottom-up process 'yet to be understood'. The work of Collins et al. [20] has sought to carefully integrate the conceptual thinking of this approach. However, as always, the real hurdles are in the engineering details. As thermodynamic entropy is meant to be increasing elsewhere (according to the maximum entropy principle [13]), so is organisational structure meant to be emerging locally (with low entropy). The massive difficulty which Wicken rightly recognised is that it has never been shown that there is any known process where protein machines can be produced spontaneously (abiogenesis). Furthermore, even with existing machines present, there has never been a case shown of bringing into being a specifically new functional machine (that is one that was not there already or latently coded for in the DNA template) from existing protein/nucleotide machinery. This line of thought has reached the same effective end-game as the previous materialistic arguments, since although these authors recognise the non-material nature of information, they are still seeking to argue for a 'bottom-up' approach to the transfer from logical reduced entropy to biological machinery.

The formidable obstacles to this proposition lie in the very nature of DNA. For example, it has been shown that DNA has the immense capability of in situ repair work. There are enzymes which are specifically assigned to nucleotide excision repair - they recognise wrongly paired bases in the DNA nucleotides [Adenine (A), Thymine (T), Cytosine (C) and Guanine (G)] connecting the two deoxyribose sugar-phosphate strands. This means that mutations are generally corrected (see, for example, the papers by Jackson [91] and de Laat et al. [92]), so that even if speciation does occur due to slight modifications and adaptations of the phylogeny, any large departures in the genetic information would be acted against by the DNA's own repair factory. Mutations do not increase the basic information content - rather the reverse is true.

At the molecular level, the laws of thermodynamics do not permit step changes in the biochemical machinery set up for a particular function performed by the cells of living organisms. Thus, random mutations always have the effect of increasing the disorder (or what can be defined as logical entropy) of any particular system, and consequently decreasing the information content.

What is evident is that the initial information content in DNA and living proteins rather than being small must in fact be large, and is in fact vital for any process to work to begin with. The issue of functional complexity and information is considered exhaustively by Meyer [93, 94] who argues that the neo-Darwinist model cannot explain all the appearances of design in biology. Even within the neo-Darwinist camp the evidence of convergence (similarity) in the suggested evolutionary development of disparate phylogeny has caused some writers such as Conway Morris [95] to consider 'channelling' of evolution. Such thinking appears to be a tacit admission of a teleological influence. The evidence indicates that functional phenotypical information does not increase by natural selection operating on random changes. 
5.3 'Top-down' approach: software and information - machines constrained by functional software

The third approach regards the information as constraining the local thermodynamics. It is clearly recognised when applying engineering principles to biological structures that in nature the vast use of information systems (see Vincent et al. [96]) with small energy usage is in great contrast to the relatively large use of energy in man-made structures with cumbersome and inefficient machinery. The field of biomimetics has opened up a whole new field of possibilities precisely because nature has such vastly superior technology. But to understand this as spontaneously arising in a bottom-up approach is to miss the key role of information constraining the local thermodynamics. This is what we now consider in this section - the 'top-down' approach.

Gitt [97] and Goonatilake [98] have shown that the Shannon information concept is not really the main contributor to real functional complexity, since this carries no concept of function and purpose (termed 'apobetics' in Gitt's work [97]; and 'teleonomy' in the work of Wilder-Smith [99]). This is essential to any real information exchange in any working system. Consequently, to define complexity as a gradual seepage in of 'negative entropy' is predicated on the notion that functional information can gradually increase from a random state. However, in reality, this type of phenotype information is not defined in the coded sequence itself (such as the DNA nucleotide sequence of Fig. 3). This point of course is recognised by Maynard Smith [63] and many other evolutionary scientists but they still seek to maintain a causal build up of phenotype information from mutations to the genotype. Rather the paradigm needs to be changed, and Gitt [97] has shown that the information is defined as five levels of signal statistics: the Shannon level, code (syntax), expression (i.e. message at the semantic level), expected action (pragmatics) and intended result (apobetics). To summarise just two of these levels succinctly, the code used is not defined by the material it orders, and the expression (message) is not defined by the code it uses. Gitt argues that information has to be thought of as a third fundamental quantity which cannot be defined in terms of matter and energy. This is rarely referred to in the literature from the evolutionary perspective. But some are certainly aware of the issue. Goonatilake [98], as stated earlier, realised that the Shannon information concept is not the right tool to attempt to define the concept of functional complexity. Nevertheless, she still maintains a bottom-up approach with the idea of increase of information flow into the earth over billions of years [100]. Brooks and Wiley [101] attempt to quantify this in a model of evolutionary speciation. Information 'gain' is presumed from a bifurcating tree of life. Perhaps the issue these authors have not considered is that Mendelian genetics actually suggests the loss of diversity under natural selection and speciation $[15,58]$. Ji [102] is also aware of the concept of information being more than the Shannon concept, and in his approach introduces the idea of 'gnergy' which is supposed to manifest itself as matter, energy, information or life. This somewhat surrealist view proposes an interplay between all of these quantities, and, as mentioned in the introduction, Davies [17] has the view that gravity can bring ordered structures into being. It is proposed by $\mathrm{Ji}$ [102] that just as mass and energy can be transformed by nuclear fission, so also information can emerge from matter.

This we refute in this paper, for although information can be formed using matter and energy [this is always being done in any modern information (computer) system], this is not done as an inherent property of the matter or energy available. What has been stated by Gitt [97] earlier is a very important concept, since it shows that all these approaches are still missing a vital concept; that is, that information is never actually defined by energy and matter, though for expression in the real world, it will necessarily need to sit upon a material/energetic substrate of some kind. At the fundamental level in the living cell, information is not actually defined by the free energy 
levels which operate the basic code in living systems (the DNA nucleotide bonds). The code is not a property of the matter itself but is a reflection of intelligence (just like a computer software code). At the next level Gitt's work draws attention to the fact that neither is the information defined by the code since the message transmitted could just as well have been transmitted using a different code. Again, this stresses that the information reflects imposed intelligence which is neither matter nor energy.

Therefore, we propose a different treatment which quantifies the effect of functional information in a system. This approach recognises Gitt's important deductions concerning real information systems being impossible to define in terms of matter and energy alone. However, we propose one can recognise the effect of machines/information systems (i.e. teleonomy) being present in exactly the same way as a digitally controlled machine (i.e. a computer) is operated by software. The high level program controls a set of electronic switches on a micro chip which are set in a certain predefined pattern. Thus, the logical entropy $d S_{\text {logical }}$ (the switching of the micro chip in the analogy) rather than being the source of the information should be thought of as the effect of information carrying systems. For a pure materialist there may be a natural reticence to adopting such an approach, but the evidence of the thermodynamics of living systems supports this. One important prediction from such a thesis is that because the thermodynamics do not actually define the information (rather the information constrains the thermodynamics), then it follows that the dissipative laws of thermodynamics will eventually destroy the substrate on which the information is sitting - and of course this is exactly what does happen. Thermodynamically, the living organism breaks down. Mutations occur and the information fades. This happens with non-digital information systems such as paper and ink, painting, etc. The information is in danger of being lost because of the inexorable action of the principle of the second law of thermodynamics. Rather than information increasing with time, the noise level increases as the order in the material substrate breaks up. Without the addition of outside intelligence, raw matter and energy will not produce auto organisation and machinery. This latter assertion is actually repeatedly borne out by experimental observation - new machinery requires intelligence. And intelligence in biological systems is from the non-material instructions of DNA.

To quote Wilder-Smith [103], (but note that in his original, his word 'closed' should read 'isolated' so in the interest of consistency we have corrected this here)

raw matter within an isolated system, plus a teleonomic machine, might yield auto-organisation derived from endogenous [that which comes from within] energy. Raw matter within a nonisolated system, plus a teleonomic machine may yield auto-organisation derived from endogenous and/or exogenous [that which comes from without] energy. Within both isolated and non-isolated systems, however, a mechanism (machine, teleonomy, know-how) is essential if any auto-organisation is to result.

The quote from Wilder-Smith is best summarised by the negative and positive statements:

$$
\text { Raw matter }+ \text { energy } \neq \text { auto-organisation, }
$$

and

$$
\text { Raw matter }+ \text { energy }+ \text { information }=\text { organisation } .
$$

It is the information within the structure which enables a non-equilibrium chemistry to be maintained, such that low logical entropy $\left(\Delta s_{\text {logical }}\right)$ is added to the fundamental molecular structure. 
Under this approach we can now go further to define information very specifically. We propose the following definition:

Definition: Information is not created by purely material processes and carries (in coded form or otherwise) intelligence.

But though information is not matter and not energy, it nevertheless always has an effect on the local thermodynamics of any working system. We thus propose the following:

Proposition: Where information interacts with matter, it always uses a machine.

Recall in Section 4.1 we defined a machine as 'a functional device which uses energy' and that other processes may use a machine but not necessarily carry information, so this is not a complete definition, on its own, of information. However, if information is present, it will have a local thermodynamic effect. The consequence of this proposition is that the effect of information can be measured in the local thermodynamics, even though the actual teleological content may not be possible to quantify.

"Significantly Polanyi [104] stated back in 1968 that

As the arrangement of a printed page is extraneous to the chemistry of the printed page, so is the base sequence in a DNA molecule extraneous to the chemical forces at work in the DNA molecule. It is the physical indeterminacy of the sequence that produces the improbability of occurrence of any particular sequence and thereby enables it to have a meaning - meaning that has a mathematically determinate information content.

Polanyi here recognised meaning as transcendent to matter and codes, though he falls back on Shannon ideas to try and measure the information, whereas we observed in section 5.1.2 that the notion of Shannon entropy is not a true metric for measuring phenotypic possibilities.

The important principle that this leads to is that the presence of information is the cause of lowered logical entropy in a given system, rather than the consequence. In living systems the principle is always that the information is transcendent to, but using raised free energy chemical bonding sites. Applying this principle to man-made and living systems provides an inherent consistency in engineering principle which fits the observations far better than the 'bottom-up' approach.

There are a number of useful examples which bring this proposition into focus.

\subsubsection{Example: letters and paper}

Words and letters carry information. There is a difference locally in the paper and inked parts of the paper. Eventually, there will be a decay process, locally. Though tiny, there is a difference in the energy levels (absorbed and reflected energy levels) of the inked paper and the paper round it, due to the surface pigment. Clearly, one could randomly put ink on the paper with meaningless marks, so that this (thermodynamics) is not a sufficient condition to ascertain real information. It is the apobetics (purpose) in information which is a vital ingredient that Gitt's work [97] exposed. Nevertheless where information is present it does have a thermodynamic effect.

\subsubsection{Computer software}

Computer software is perhaps the best example of information since this is a close parallel to living systems. Software has no mass - the mass of a CD/DVD is no different with or without information present. Moreover programs and software are not defined by matter or energy but nevertheless the instructions do have an effect on what they are inscribed, and on the computer chip once loaded into memory. It is essential to have software instructions, as we all experience, for modern digital computers to work. As stated earlier, the program software controls a set of electronic micro switches - it 
gives them all small but significant changes in local free energy. Thus, the effect of information is to have a local thermodynamic effect - in that case it raises the local free energies. It constrains the local thermodynamics to be in a certain pattern.

\subsubsection{DNA coding}

According to this definition of information, this now makes a great deal of sense regarding the observations concerning the DNA code. The whole set of instructions, though certainly suffering in the present day from mutations, nevertheless, is essentially reflecting a sequence of vast numbers of instructions, consistent with intelligent origin. Seeking to find a 'bottom-up' approach which tries to insist on a mechanism of setting up thousands of free energy levels all with no initial purpose leads to the severe difficulties we discussed in Sections 4.3.2, 5.1 and 5.2 concerning the impossibility of bringing together a suite of free energy levels in the molecular machinery. By first acknowledging that the basic coding is the cause (and thus reflects an initial purpose) rather than the consequence, it gives a much better paradigm for understanding the molecular machinery which is now consistent with known thermodynamic principles. It also encourages genetic research into piecing together the correction of key genetic disease machinery by knowing that there is an original perfect genetic blueprint.

\subsubsection{Music}

Music carries information. This type of information may be subjective, but there are patterns of notes conveyed to an instrument. The information (music) is not interacting with matter and energy until the violin or piano or flute and so on are made to generate local thermodynamic differences this time with air - through compression and rarefaction, etc. The thermodynamic effect is essential to convey the information.

\subsubsection{Painting}

The concept even flows through to painting and drawing and makes sense of much that we know as aesthetic information. The picture cannot really be defined by the thermodynamics. But it is the differences locally of pigments held by surfaces that give the impression of a picture. The artist cannot convey the image unless locally the image interacts with the matter and energy of the paint/ canvas system.

\section{CONCLUSIONS - LOCAL THERMODYNAMICS CONSTRAINED BY FUNCTIONAL INFORMATION}

It has often been asserted that the logical entropy of a non-isolated system could reduce, and thereby new information could occur at the expense of increasing entropy elsewhere, and without the involvement of intelligence. In this paper, we have sought to refute this claim on the basis that this is not a sufficient condition to achieve a rise in local order. One always needs a machine in place to make use of an influx of new energy and a new machine inevitably involves the systematic raising of free energies for such machines to work. Intelligence is a pre-requisite.

The defining of information is a key issue in the origins debate, since terms such as 'advance' and 'simple to complex' have little direct meaning at the biochemical level. Intuitive reasoning presupposes the scientific paradigm adopted by the authors of such statements, and the discussions concerning origins are fundamentally to do with those paradigms. Consequently, the biochemical arguments are always going to be a matter of contention because of the root issues at stake.

In this paper, we have considered the fundamental definition of what is a machine and then moved on to consider the definition of information in the context of how information interacts with the 
physical world. We have proposed that at the fundamental level, rather than regarding negative entropy as being a source of information, it is far more self-consistent to regard the information defined in terms of a source from which negative logical entropy is derived at the molecular level, such that local free energy levels are maintained and biological structures can work.

Asserting that the local entropy of a non-isolated system could reduce through chance exchanges of that system with its environment is not an adequate answer to the origin of new machinery and information. By considering the Gibbs free energy connecting two possible states, it is evident that this assertion involves thermodynamic hurdles which demand effectively a different physics. The building of molecular machinery only takes place when existing information is already inherent in the system the information comes first before the machinery. In a non-isolated system, energy (such as from the sun) may increase the local temperature difference (and thus increase the potential for useful work that can be done locally), but without a machine (that is, a device which is made or programmed to use the available energy), there is still no possibility of the self-organisation of matter without an information system present. There has to be previously written information or order (often termed 'teleonomy' [103]) for passive, non-living chemicals to respond and become active. Thus, the following summary statement applies to all known systems:

$$
\begin{gathered}
\text { Energy }+ \text { Information (or teleonomy) } \rightarrow \text { Locally reduced entropy } \\
\text { (and increase in order)) }
\end{gathered}
$$

with the corollary:

$$
\text { Matter and energy alone } \nRightarrow \text { Decrease in entropy (and increase in order) }
$$

Another way of saying this is that for a non-isolated system, energy must be directed to be of any use.

We have argued that the free energy of the structures in living systems mitigates strongly against the thesis that machines can come about spontaneously, and that for living systems, rather than regarding negative entropy as a quantity generated within, one should regard the information as being the cause and the logical entropy reduction as being the result. Information is not matter and not energy but is a fundamental part of reality. Moreover, intelligence precedes both machines and information systems, and wherever information interacts with the physical world, there is a measurable thermodynamic effect.

Of course, the implication of this paper is that it supports the so-called intelligent design thesis that an intelligent designer is needed to put the information into the biological system. Even though many modern scientists find this conclusion unacceptable for philosophical reasons, it is, nevertheless, a logical outcome of the thermodynamic arguments as presented in this paper.

\section{ACKNOWLEDGEMENTS}

The author wishes to thank the referees for their careful and painstaking work in reviewing this paper. He is also very grateful to the editors Professors Carlos Brebbia and Stuart Burgess for their guidance and editorial work. This paper has been vastly improved from the original submission as a result of the labours of reviewers and editors.

\section{REFERENCES}

[1] Watson, J.D. \& Crick, F.H.C., Molecular structure of nucleic acids. Nature, 171, pp. 737-738, 1953. doi:10.1038/171737a0

[2] McIntosh, A.C., Functional information and entropy in living systems. Design and Nature III Third International Conference on Design \& Nature. Comparing Design in Nature with Science and Engineering, 24th-26th May 2006, Vol. 87 of WIT Transactions on Ecology and 
the Environment, ed. C.A. Brebbia, WIT Press, Wessex Institute of Technology: New Forest, 2006, pp. 115-126. ISBN: 1-84564-166-3.

[3] Nicolis, G. \& Prigogine, I., Exploring Complexity: An Introduction, W.H. Freeman: New York, 1989. And the useful web site where these matters of non-equilibrium thermodynamics are discussed: http://www.schuelers.com/ChaosPsyche/part_1_9.htm (accessed 2006).

[4] Kondepudi, D. \& Prigogine, I., Modern Thermodynamics - from Heat Engines to Dissipative Structures, Wiley, 1999.

[5] Prigogine, I., Nicolis, G. \& Babloyantz, A., Thermodynamics of evolution (I). Physics Today, 25(11), pp. 23-28, 1972. doi:10.1063/1.3071090

[6] Prigogine, I., Nicolis, G. \& Babloyantz, A., Thermodynamics of evolution (II). Physics Today, 25(12), pp. 28-44, 1972. doi:10.1063/1.3071140

[7] Mayr, E., What Evolution is, Weidenfeld and Nicolson - Orion Publishing: London, 2001.

[8] Maynard Smith, J. \& Szathmary, E., The Origins of Life, Oxford University Press: Oxford, New York, 1999.

[9] Ball, P., The Self-made Tapestry - Pattern Formation in Nature, Oxford University Press: Oxford, New York, 2001.

[10] Wicken, J.S., Evolution, Thermodynamics, and Information: Extending the Darwinian Program, Oxford University Press, 1987.

[11] Kauffman, S.A., The origins of life: a new view (Chapter 7). Origins of Order-Self-organisation and Selection in Evolution, Oxford University Press, pp. 287-341, 1993.

[12] Brooks, D.R. \& Wiley, E.O., Evolution as Entropy: Towards a Unified Theory of Biology, University of Chicago Press: Chicago and London, 1986.

[13] Martyushev, L.M. \& Seleznev, V.D., Maximum entropy principle in physics, chemistry and biology. Physics Reports, 426, pp. 1-45, 2006. doi:10.1016/j.physrep.2005.12.001

[14] Shannon, C.E., The mathematical theory of communication. The Bell System Technical Journal, 27, pp. 379-423, 623-656, 1948.

[15] Dawkins, R., The information challenge (Chapter 2.3). A Devil's Chaplain; Selected Essays by Richard Dawkins, ed. Latha Menon, Phoenix, pp. 107-122, 2003.

[16] Goonatilake, S., The Evolution of Information: Lineages in Gene, Culture and Artefact, Pinter: London, 1991.

[17] Davies, P., The Fifth Miracle - the Search for the Origin of Life, Penguin: London, 1999.

[18] Murray, J.D., Mathematical Biology. II: Spatial Models and Biomedical Applications, 3rd edn, Springer-Verlag: New York, 2000.

[19] Coveney, P. \& Highfield, R., The Arrow of Time, Harper Collins: London, 1991.

[20] Collins, M.W., Stasiek, J.A. \& Mikielewicz, J., The laws of thermodynamics: entropy, free energy, information and complexity (Chapter 5). Design and Information in Biology - From Molecules to Systems, eds J.A. Bryant, M.A. Atherton \& M.W. Collins, WIT Press, Wessex Institute of Technology: New Forest, pp. 127-143, 2007. ISBN: 978-185312-856-0.

[21] Chaisson, E.J., The cosmic environment for the growth of complexity. Biosystems, 46, pp. 13-19, 1998. doi:10.1016/S0303-2647(97)00076-2

[22] Penrose, R., The Emperor's New Mind, Vintage: London, 1990.

[23] Le Fanu, J., Why Us? How Science Rediscovered the Mystery of Ourselves, Harper Press: London, 2009.

[24] Thomson, W., On the dynamical theory of heat. Transactions of the Royal Society of Edinburgh, 1851; Philosophical Magazine IV, 1852. Quotes from Mathematical and Physical Papers, i, art. XLVIII, p. 174. Specific quote here from paragraph 12. 
[25] Joule, J. P., On the calorific effects of magneto-electricity and the mechanical value of heat British Association, 1843, Philosophical Magazine, 1843.

[26] Thomson, W., 'On the Dynamical Theory of Heat', Transactions of the Royal Society of Edinburgh, 1851, and Philosophical Magazine IV. 1852. Quotes from Mathematical and Physical Papers, i, art. XLVIII, p. 174. Specific quote here end of paragraph 13.

[27] Thomson, W., On a universal tendency in nature to the dissipation of mechanical energy'. Proc. R. Soc. Edinburgh, 1852, Philosophical Magazine, 1852. This paper takes the quotes from Mathematical and Physical Papers, i, art. 59, pp. 511.

[28] Thompson, S.P., Life of Lord Kelvin, Macmillan: London, pp. 290-291, 1910.

[29] Rogers, G. \& Mayhew, Y., Engineering Thermodynamics, Work and Heat Transfer, Prentice Hall, Longman: Harlow, 1992.

[30] Styer, D.F., Entropy and evolution. American Journal of Physics, 76(11), 1031-1033, 2008. doi: $10.1119 / 1.2973046$

[31] Bunn, E.F., Evolution and the second law of thermodynamics. American Journal of Physics, 2009, in press. doi:10.1119/1.3119513

[32] Ji, S., Biocybernetics: a machine theory of biology. Molecular Theories of Life and Death, ed. S. Ji, Rutgers University Press: New Brunswick, New Jersey, 1991.

[33] I am indebted here to a private communication with Professor Werner Gitt, who for many years was Head of the Department of Information Technology.at the German Federal Institute of Physics and Technology (Physikalisch-Technische Bundesanstalt, Braunschweig).

[34] Rey, A.D., Nonlinear actuator model for flexoelectric membranes. Int. J. Des. Nat. 3(1), pp. 28-38, 2008.

[35] Richard Dawkins, on the programme BBC Ulster "Sunday sequence" 10th Dec 2006 in conversation with the author, sought to maintain that fresh energy from the Sun would resolve the dilemma of setting up the first DNA (abiogenesis). But such functionality cannot take place spontaneously because $\Delta G>0$. Furthermore even after abiogenesis, [if ever this could occur], entirely new machines could not arise unless again the local Gibbs free energy $\Delta \mathrm{G}>0$ for the new machine. This will not happen by an arbitrary appeal to 'any machines will do'. The machine necessary will be very specific - e.g the machine for attaching amino acids (where again the bonds to have positive Gibbs free energy) to make proteins - the ribosome - is very specific, and has to be in place to raise the local Gibbs free energy to the appropriate level in the protein chemistry as the amino acids join together.

[36] Thaxton, C.B., Bradley, W.L. \& Olsen, R.L., Thermodynamics and the origin of life (Chapter 8). The Mystery of Life's Origin, Lewis and Stanley: Dallas, pp. 127-143, 1992.

[37] Wicken, J.S., op. cit., Evolution, Thermodynamics, and Information, p. 71.

[38] Betina, S., Goffrini, P., Ferrero, I. \& Wesolowski-Louvel, M., RAG4 gene encodes a glucose sensor in Kluyveromyces lactis. Genetics, 158, pp. 541-548, 2001.

[39] Leakey, R. \& Lewin, R., Origins, Macdonald and Jane's: London, 1977.

[40] Attenborough, D., The Living Planet, (Film), BBC: London, 1985. Well known for his exquisitely produced documentaries, he has produced many films like this which more than anything else have popularised the evolutionary viewpoint in the UK.

[41] Cairns-Smith, A.G., Seven Clues to the Origin of Life. A Scientific Detective Story, Cambridge University Press, 1985.

[42] Cruywagen, G.C., Maini, P.K. \& Murray, J.D., Sequential pattern formation in a model for skin morphogenesis. IMA Journal of Mathematics Applied in Medicine and Biology, $\mathbf{9}$, pp. 227-248, 1992. doi:10.1093/imammb/9.4.227 
[43] Cruywagen, G.C., Maini, P.K. \& Murray, J.D., Sequential and synchronous skin pattern formation (Chapter 8). Experimental and Theoretical Advances in Biological Pattern Formation, ed. H.G. Othmer, Plenum: New York, 1993.

[44] Maynard Smith, J. \& Szathmary, E., op. cit., The Origins of Life, p. 116.

[45] Tramontano, A., Problem 4 protein structure prediction (Chapter 4). The Ten Most Wanted Solutions in Protein Bioinformatics, Chapman and Hall, pp. 69-88, 2005.

[46] Tramontano, A., op. cit., The Ten Most Wanted Solutions, p. 69.

[47] Birney, E. et. al., Identification and analysis of functional elements in $1 \%$ of the humangenome by the ENCODE pilot project. Nature, 447, pp. 799-816, 2007. doi:10.1038/nature05874

[48] Schneider, T.D., Evolution of biological information. Nucleic Acids Research, 28(14), pp. 2794-2799, 2000. doi:10.1093/nar/28.14.2794

[49] Truman, R., The problem of information for the theory of evolution. True Origin Archive, http://www.trueorigin.org/schneider.asp, 2001, accessed 30th May 2008.

[50] Truman, R., op. cit., The problem of information, section 'Background'.

[51] Kimura, M., Natural selection as the process of accumulating genetic information in adaptive evolution. Genetic Research, 2, pp. 127-140, 1961. doi:10.1017/S0016672300000616

[52] Kimura, M., Evolutionary rate at the molecular level. Nature, 217, pp. 624-626, 1968. doi: $10.1038 / 217624 \mathrm{a} 0$

[53] Kimura, M., The rate of molecular evolution considered from the standpoint of population genetics. Proc. Natl. Acad. Sci. USA, 63, pp. 1181-1188, 1969. doi:10.1073/pnas.63.4.1181

[54] Ohta, T. \& Kimura, M., Statistical analysis of the base composition of genes using data on the amino acid composition of proteins. Genetics, 64, pp. 387-395, 1970.

[55] Ohta, T. \& Kimura, M., Genetic load due to mutations with very small effects. Japanese Journal of Genetics, 46, pp. 393-401, 1971. doi:10.1266/jjg.46.393

[56] Kimura, M., Neutral Theory of Molecular Evolution, Cambridge University Press: Cambridge, New York, pp. 30-31, 1983. doi:10.1017/CBO9780511623486

[57] Kimura, M., Model of effectively neutral mutations in which selective constraint is incorporated. Proc. Natl. Acad. Sci., 76(7), pp. 3440-3444, 1979. See particularly Fig. 1 on p. 3441.

[58] Sanford, J.C., Are random mutations good? (Chapter 2). Genetic Entropy and the Mystery of the Genome, Ivan Press, pp. 15-32, 2005.

[59] Kim, J.T., Martinetz, T. \& Polani, D., Bioinformatic principles underlying the information content of transcription factor binding sites. J. Theor. Biol., 220, pp. 529-544, 2003. doi:10.1006/ jtbi.2003.3153

[60] Kimura, M., Neutral Theory of Molecular Evolution, Cambridge University Press: Cambridge, New York, p. 26, 1983. doi:10.1017/CBO9780511623486

[61] Haldane, J.B.S., The cost of natural selection. J. Genetics, 55, pp. 511-524, 1957. doi:10.1007/ BF02984069

[62] Sanford, J.C., op. cit., Genetic Entropy, pp. 78-79.

[63] Maynard Smith, The idea of information in Biology. The Quarterly Review of Biology, 74(4), pp. 395-400, 1999. See p. 397, 'Genotype and Phenotype'.

[64] Joyce, G.G., RNA evolution and the origins of life. Nature, 338, pp. 217-224, 1989. doi:10.1038/338217a0

[65] Muller, H.J., The gene material as the initiator and organizing basis of life. American Naturalist, 100, pp. 493-517, 1966. doi:10.1086/282445

[66] Trevors, J.T. \& Abel, D.L., Chance and necessity do not explain the origin of life. Cell Biology International, 28, pp. 729-739, 2004. doi:10.1016/j.cellbi.2004.06.006 
[67] Behe, M., Darwin's Black Box, Simon and Schuster, Free Press: New York, pp. 69-73, 1996.

[68] Pallen, M.J. \& Matzke, N.J., From the origin of species to the origin of bacterial flagella. Nature Reviews Microbiology, 4, pp. 784-790, 2006. doi:10.1038/nrmicro1493

[69] Yockey, H., Information Theory and Molecular Biology, Cambridge University Press, 1992.

[70] Demetrius, L., Directionality principles in thermodynamics and evolution. Proc. Natl. Acad. Sci. USA, 94, pp. 3491-3498, 1997. doi:10.1073/pnas.94.8.3491

[71] Wiley, E.O. \& Brooks, D.R., Victims of history - a non-equilibrium approach to evolution. Systematic Zoology, 31(1), pp. 1-24, 1988. doi:10.2307/2413410

[72] Brooks, D.R. \& Wiley, E.O., Nonequilibrium thermodynamics and evolution: responses to Bookstein and to Wicken. Systematic Zoology, 34(1), pp. 89-97, 1985. See p. 96 first column in particular.

[73] Davies, P., op. cit., The fifth miracle, p. 122.

[74] Wicken, J.S., op. cit., Evolution, Thermodynamics, and Information, p. 78.

[75] Wicken, J.S., op. cit., Evolution, Thermodynamics, and Information, p. 124.

[76] Wicken, J.S., op. cit., Evolution, Thermodynamics, and Information, p. 104.

[77] Collier, J., Out of equilibrium: new approaches to biological and social change. Biology and Philosophy, 8, pp. 445-455, 1993. doi:10.1007/BF00857690

[78] Kauffman, S.A., op. cit., Origins of Order, p. 287.

[79] Kauffman, S.A., op. cit., Origins of Order, pp. 318-322.

[80] Kauffman, S.A., op. cit., Origins of Order, p. 319.

[81] Miller, S.L., Production of amino acids under possible primitive earth conditions. Science, 117, pp. 528, 1953.

[82] Horgan, J., In the beginning. Scientific American, 264, pp. 116-125, 1991. Section concerning Stanley Miller, p. 117.

[83] Kauffman, S.A., Random grammars: models of functional integration and transformation (Chapter 10). Origins of Order - Self-organisation and Selection in Evolution, Oxford University Press, pp. 369-404, 1993.

[84] Kauffman, S.A., op. cit. Origins of Order, pp. 370-372.

[85] Kauffman, S.A., op. cit. Origins of Order, p. 289.

[86] Ulanowicz, R.E., 'Natural life beyond Newton and Darwin', Templeton Press, 2009, See foreword by Stuart Kauffman.

[87] Wicken, J.S., The generation of complexity in evolution: a thermodynamic and informationtheoretical discussion. Journal of Theoretical Biology, 77, pp. 349-365 and p. 349, 1979. doi:10.1186/1742-4682-2-29

[88] Abel, D.L. \& Trevors, J.T., Three subsets of sequence complexity and their relevance to biolpolymeric information. Theoretical Biology and Medical Modelling, 2, p. 29, 2005.

[89] Abel, D.L., The capabilities of chaos and complexity. Int. J. Mol. Sci., 10, pp. 247-291, 2009. doi:10.3390/ijms 10010247

[90] Cambel, A.B., Applied Chaos Theory: a Paradigm for Complexity, Academic Press: Boston, 1993.

[91] Jackson, S.P., Sensing and repairing DNA double strand breaks. Carcinogenesis, 23(5), pp. 687-696, 2002. doi:10.1093/carcin/23.5.687

[92] de Laat, W.L., Jaspers, N.G.J. \& Hoeijmakers, J.H.J., Molecular mechanism of nucleotide excision repair. Genes and Development, 13(7), pp. 768-785, 1999. doi:10.1101/gad.13.7.768

[93] Meyer, S.C., The origin of biological information and the higher taxonomic categories. Proceedings of the Biological Society of Washington, 117(2), pp. 213-239, 2004. 
[94] Meyer, S.C., Signature in the Cell: DNA and the Evidence for Intelligent Design, HarperOne: San Francisco, 2009.

[95] Conway Morris, S., Evolution: bringing molecules into the fold. Cell, 100, pp. 1-11, 2000. doi:10.1016/S0092-8674(00)81679-7

[96] Vincent, J.F.V., Bogatyreva, O.A., Bogatyrev, N.R., Bowyer, A. \& Pahl, A.K., Biomimetics: its practice and theory. Journal of the Royal Society Interface, 3, pp. 471-482, 2006. In particular see discussion on p. 477 of nature's vast resources of information and small energy useage by comparison with man made technology.

[97] Gitt, W., Information: the third fundamental quantity. Siemens Review, 56, Part 6, pp. 36-41, 1989.

[98] Goonatilake, S., op. cit. The evolution of information: lineages in gene, culture and artefact, p. 163.

[99] Wilder-Smith, A.E., The Natural Sciences Know Nothing of Evolution, Master Books: San Diego, California, p. 59, 1981.

[100] Goonatilake, S., op. cit. The evolution of information: lineages in gene, culture and artefact, figure 9.3, p. 133.

[101] Brooks, D.R. \& Wiley, E.O., op. cit. Evolution as entropy: towards a unified theory of biology, pp. 340-345.

[102] Ji, S., op. cit. Molecular Theories of Life and Death, figure 1.A5, p. 234.

[103] Wilder-Smith, A.E., op. cit., The Natural Sciences Know Nothing of Evolution. See particularly chapter 4 "The Genesis of Biological Information". Bracketed material added. Wilder Smith in the original book used the term 'closed' when strictly the thermodynamic concept he was referring to was an 'isolated' system. Therefore in the interest of consistency we have corrected this terminology here.

[104] Polanyi, M. Life's irreducible structure, Science 160(3834), 1308-1312, 1968, p. 1309. 